\title{
Artemisia annua Growing Wild in Romania-A Metabolite Profile Approach to Target a Drug Delivery System Based on Magnetite Nanoparticles
}

\author{
Adina-Elena Segneanu ${ }^{1, *} \mathbb{0}$, Catalin Nicolae Marin ${ }^{1}$, Ioan Ovidiu-Florin Ghirlea ${ }^{2}$, Catalin Vladut Ionut Feier ${ }^{2}$, \\ Cornelia Muntean ${ }^{3}$ (D) and Ioan Grozescu ${ }^{3}$ \\ 1 Faculty of Physics, West University of Timisoara, Blvd. V. Parvan 4, 300223 Timisoara, Romania; \\ catalin.marin@e-uvt.ro \\ 2 Faculty of Medicine, Victor Babes University of Medicine and Pharmacy Timisoara, 2 P-ta E. Murgu, \\ 300041 Timisoara, Romania; ovidiu.ghirlea@gmail.com (I.O.-F.G.); catalinfeier10@gmail.com (C.V.I.F.) \\ 3 Faculty of Industrial Chemistry and Environmental Engineering, Polytechnic University Timisoara, \\ 2 P-ta Victoriei, 300006 Timisoara, Romania; cornelia.muntean@upt.ro (C.M.); ioangrozescu@gmail.com (I.G.) \\ * Correspondence: adina.segneanu@e-uvt.ro
}

Citation: Segneanu, A.-E.; Marin, C.N.; Ghirlea, I.O.-F.; Feier, C.V.I.; Muntean, C.; Grozescu, I. Artemisia annua Growing Wild in Romania-A Metabolite Profile Approach to Target a Drug Delivery System Based on Magnetite Nanoparticles. Plants 2021, 10, 2245. https://doi.org/10.3390/ plants10112245

Academic Editors: Ivayla

N. Dincheva, Ilian Badjakov and Bistra Galunska

Received: 18 September 2021

Accepted: 13 October 2021

Published: 21 October 2021

Publisher's Note: MDPI stays neutral with regard to jurisdictional claims in published maps and institutional affiliations.

Copyright: (c) 2021 by the authors. Licensee MDPI, Basel, Switzerland. This article is an open access article distributed under the terms and conditions of the Creative Commons Attribution (CC BY) license (https:// creativecommons.org/licenses/by/ $4.0 /)$.

\begin{abstract}
The metabolites profile of a plant is greatly influenced by geographical factors and the ecological environment. Various studies focused on artemisinin and its derivates for their antiparasitic and antitumoral effects. However, after the isolation and purification stage, their pharmaceutical potential is limited due to their low bioavailability, permeability and lifetime. The antibacterial activity of essential oils has been another topic of interest for many studies on this plant. Nevertheless, only a few studies investigate other metabolites in Artemisia annua. Considering that secondary metabolites act synergistically in a plant, the existence of other metabolites with antitumor and high immunomodulating activity is even more important. Novel nano-carrier systems obtained by loading herbs into magnetic nanoparticles ensures the increase in the antitumor effect, but also, overcoming the barriers related to permeability, localization. This study reported the first complete metabolic profile from wild grown Romanian Artemisia annua. A total of 103 metabolites were identified under mass spectra (MS) positive mode from 13 secondary metabolite categories: amino acids, terpenoids, steroids, coumarins, flavonoids, organic acids, fatty acids, phenolic acids, carbohydrates, glycosides, aldehydes, hydrocarbons, etc. In addition, the biological activity of each class of metabolites was discussed. We further developed a simple and inexpensive nano-carrier system with the intention to capitalize on the beneficial properties of both components. Evaluation of the nano-carrier system's morpho-structural and magnetic properties was performed.
\end{abstract}

Keywords: secondary metabolites; GC-MS; mass-spectra; bioactive compounds; nano-carrier system; magnetic nanoparticles

\section{Introduction}

Romanian phytotherapy has an ancient and very rich tradition based on a very wide diversity of medicinal plants. Thus, in the spontaneous flora of Romania there are about 800 species of medicinal plants. Additionally, plants of the genus Artemisia (Asteraceae) form part of this phytopharmacological treasure.

Artemisia annua (common name: wormwood or năfurica in Romanian) is one of the ancient healing plants recognized in traditional medicine from Europe and Asia. Romanian traditional medicine has exploited its therapeutic properties: antihemorrhagic, antiseptic, antioxidant, digestive, antipyretic, immunomodulatory, antibacterial and antitumoral [1-5]. Artemisia annua is used also in Romania to prepare a digestive wine [1-5]. In traditional Asian pharmacopoeia, it was recommended, especially for the treatment of fevers and colds. 
Chemical screening of bioactive compounds isolated from this plant has highlighted the rich content in volatile compounds, terpenes, sesquiterpenes, alkaloids, flavonoids, coumarins and phenolic compounds.

Studies on biological properties of secondary metabolites isolated from extracts of Artemisia annua have confirmed its therapeutic properties (antioxidant, antidiabetic, antiviral, antitumor, immunomodulatory, antiparasitic, antibacterial and antifungal activity) described in traditional medicine [4-10].

Additionally, the latest studies in the field aim to evaluate its potential application in the prevention and treatment of SARS-CoV 19 [6-23].

In recent years, numerous studies have been conducted on the main classes of phytoconstituents (phenolic compounds, alkaloids, terpenes and volatile compounds) in sweet wormwood. Special attention was paid to the isolation and chemical synthesis of artemisinin, considered the main bioactive compound of the plant, with antiparasitic activity. This was an important step in modern research in medicinal plants and in the fight against malaria, an infectious disease that affects the lives of millions of people [6-20]. According to World Health Organization estimates, malaria killed more than 400 million people globally in 2019 alone [24].

Artemisinin is widely used for malaria treatment, and recent studies demonstrated its antitumoral activity on several cancer cell lines. However, at present, the production costs of artemisinin are high. Moreover, its low bioavailability, permeability and life-time in biological media represent the main biomedical limitations [25].

It is known that the biological activity of a plant is due to the synergistic effect of different types of phytoconstituents $[26,27]$. Therefore, the chemical screening and complete identification of bioactive components from a plant is especially important.

Recent studies have shown that the antitumor activity of the plant is not only due to artemisinin. This might be just one of the plant metabolites with high biological activity [6].

Although the plant has been studied for a long time, its chemical composition is influenced by many understudied factors, among which we only mention geographical position, climate, soil $\mathrm{pH}$ and so on [28].

For these reasons, studies on this plant are particularly important given its special therapeutic potential.

Additionally, some small peptides in the composition of this plant could be another key constituent to antitumor activity.

Currently, there are several therapeutic approaches to cancer, including drugs, genetics and anticancer peptides. The results of studies regarding peptide anticancer therapy showed that the sequence of these small anticancer peptides can include several amino acids, such as: arginine, glycine, lysine and leucine, glutamic and aspartic, tyrosine, phenylalanine, proline and protonated histidine. Moreover, recent research has showed that arginine has a key role in the function of the immune system and antitumor activity [29-31].

Nevertheless, there are very few studies that have investigated the amino acid composition of Artemisia genus [32].

Studies regarding wild Romanian Artemisia genus are few and targeted, especially on bioactive components of Artemisia absinthium [33,34]. Furthermore, regarding Romanian Artemisia annua wild plant, the present research only investigates the content of artemisinin and volatile oils [35-37].

Despite its high therapeutic potential, the chemical screening of the biologically active compounds of this medicinal plant from the spontaneous flora of Romania has not been performed yet.

On the other hand, it should be mentioned that several herbal supplements of Artemisia annua are marketed globally, which are recommended, according to the manufacturers, for malaria, arthritis and even cancer.

Recently, several cases of liver disease have been reported, especially following selfmedication with herbal supplements to manage cancer or prevent malaria $[38,39]$. 
The development of highly efficient, selective, simple and inexpensive nano-carrier systems could be an effective method to avoid these risks while ensuring the controlled intake of phytoconstituents with biological activity [40,41].

Modern drug delivery systems based on magnetic nanoparticles could easily accomplish these requirements. Moreover, the latest developments regarding magnetite nanoparticles have demonstrated their benefits and recommend their use in drug delivery and other different biomedical applications (magnetic resonance imaging, tumor therapeutic hyperthermia, etc.) [25,42].

Magnetic/superparamagnetic nanoparticles could represent a more than interesting alternative due to their advantages: their capability for local delivery and the ability to act selectively. However, the possibility of an immune response is the main drawback of these nanoparticles. Therefore, the design of new drug delivery systems based on magnetic/superparamagnetic nanoparticles for use as early detection methods and in the diagnosis, prognosis and monitoring of the evolution of the cancer treatment is required given the social impact of these diseases [25,43-49].

Recent studies have shown that nano-carriers based on magnetic nanoparticles lead to a high drug tissue permeability and retention effect and thus enhance the beneficial therapeutic effects [45-47].

To our best knowledge, this study investigates the metabolite profile of Artemisia annua grown wild in Romania for the first time. Subsequently, a simple and inexpensive nano-carrier system that capitalizes both the therapeutic properties of Artemisia annua (whole plant) and magnetic $\mathrm{Fe}_{3} \mathrm{O}_{4}$ nanoparticles was developed.

\section{Results and Discussion}

Extensive research in the field of plants and especially on those with high therapeutic potential has shown that they have a very complex composition of compounds with high biological activity that act synergistically in the body [26,27].

Additionally, a full description of a general metabolic profile for a specific herb is all the more difficult, as significant differences in secondary metabolites was reported among the same plants harvested from various geographic regions of the world. Studies in this area confirmed that the content of specific plant secondary metabolites is the result of several environmental stress factors (climate, soil and biological conditions) which directly influence plant growth, development and topography distribution [28,50-52].

The pharmacological properties of different plant secondary metabolites were extensively investigated. However, their therapeutic benefits have not been completely understood $[11,21,22]$. Plant metabolites with peptide structures are just an example of this [51].

Bioactive metabolite chemical screening of sweet wormwood (năfurica) was tentatively carried out via gas-chromatography coupled with mass spectroscopy (GC-MS) and electrospray ionization-quadrupole time-of-flight mass spectrometry (ESI-QTOF-MS) analysis.

In addition, the amino acid profile was investigated using GC-MS techniques (Figure 1). The mass spectra of components identified were determined via comparison of their retention indices and mass spectra with those of NIST/EPA/NIH, the Mass Spectral Library 2.0 data base, as well as by reviewing the literature [32,53]. 


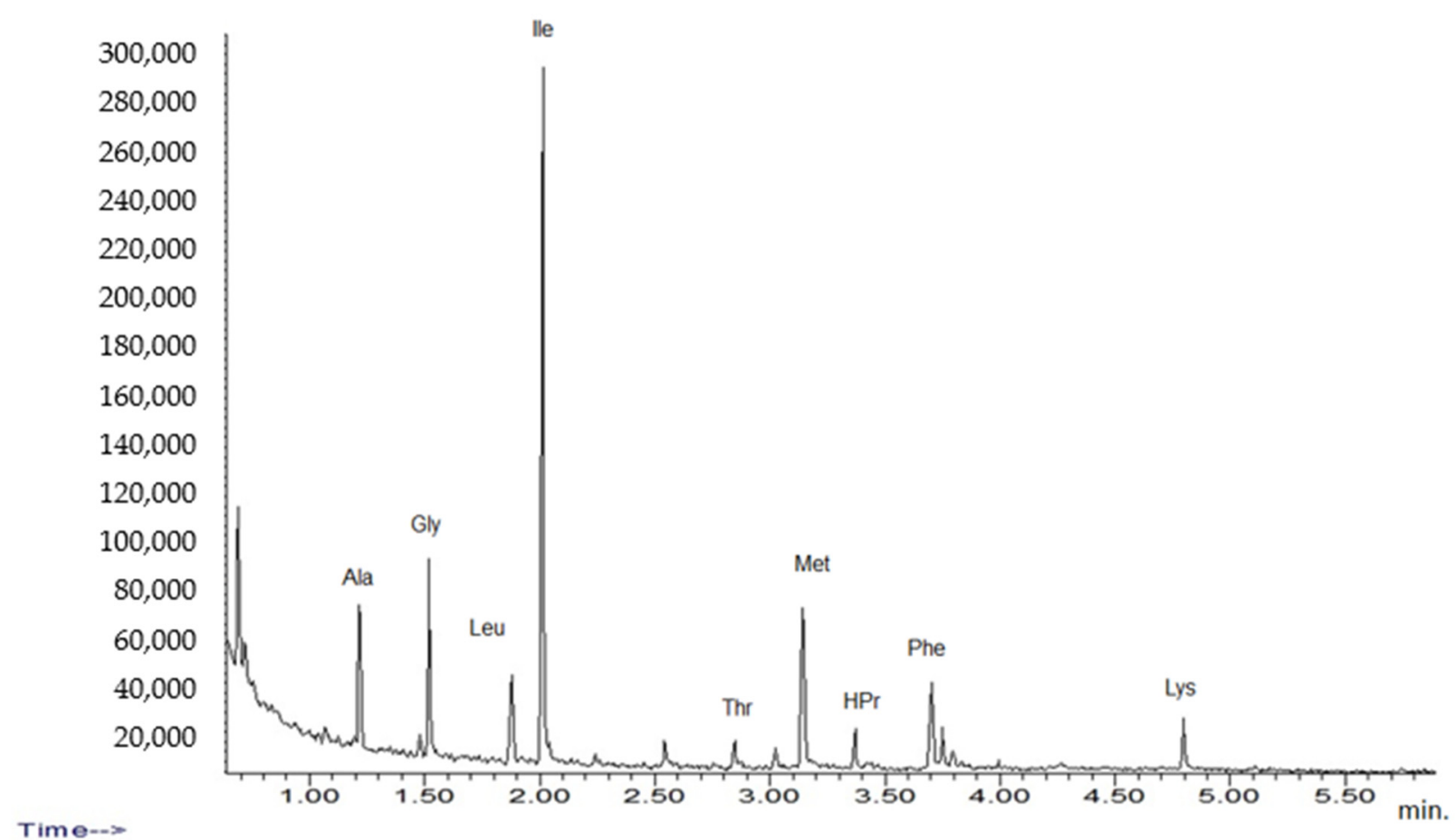

Figure 1. TIC chromatograms of GC-MS for Artemisia annua.

The results are listed in Table 1, which presents tentative amino acid identification via GC-MS corresponding to Artemisia annua sample [32,54,55].

Table 1. Main compounds identified by GC-MS analysis of plant extract.

\begin{tabular}{ccc}
\hline Proposed Structure & Abbreviation & $\begin{array}{c}\text { SIM (Selected Ion } \\
\text { Monitoring) }\end{array}$ \\
\hline Alanine & Ala & 130,70 \\
\hline Leucine & Leu & 172,86 \\
\hline Glycine & Gly & 116,74 \\
\hline Isoleucine & Ile & 172,130 \\
\hline Methionine & Met & 203,277 \\
\hline Phenylalanine & Phe & 206,190 \\
\hline Lysine & Lys & 170,128 \\
\hline Threonine & Thr & 160,101 \\
\hline 4-Hydroxyproline & HYP & 172,86 \\
\hline
\end{tabular}

\subsection{Mass Spectrometry Analysis of Romanian Artemisia annua}

The plant sample was diluted in methanol and analyzed using ESI-TOF mass spectroscopy (ESI-QTOF-MS). The plant extract sample analysis was carried out in the positive mode. The mass spectra (Figure 2) showed the presence of complex metabolite composition. A total of 103 compounds were detected and identified, which covered various chemical categories, including amino acids, sterols, terpenoids, flavonoids, coumarins, alcohols, aldehydes, glycosides, carbohydrates, fatty acids and so on, which confirmed the data reported in the literature [6-24,28,31-39,46,53-60]. Additionally, the presence of amino acids identified through GC-MS was confirmed via ESI-QTOF-MS analysis. However, only a few studies reported the amino acid profile of Artemisia annua [32]. 


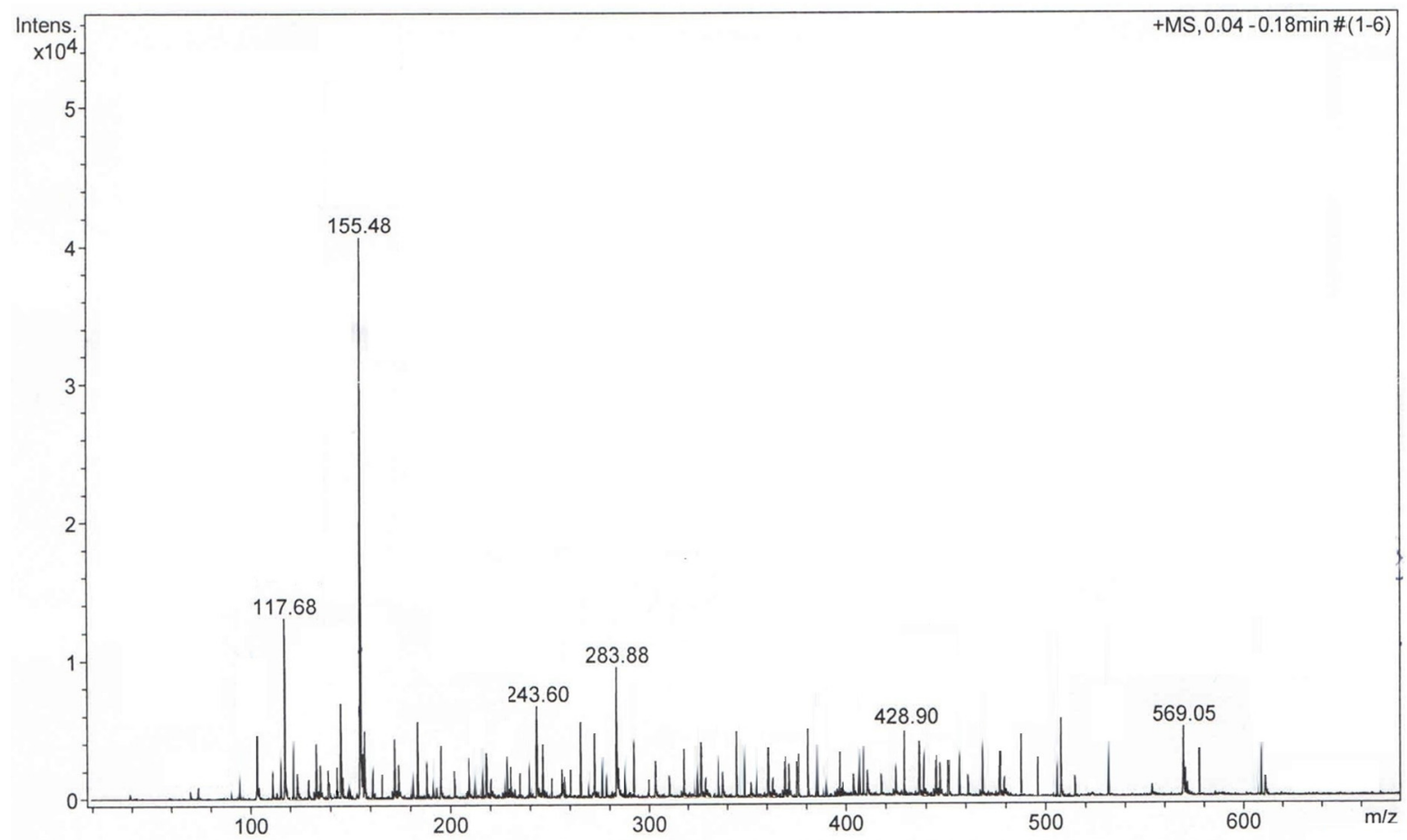

Figure 2. Positive ion mode MS-TOF of Artemisia annua sample.

The identified metabolites are listed in Table 2 and classified according to their $\mathrm{m} / \mathrm{z}$ ratio (mass-to-charge-ratio) (theoretical and measured), chemical name and formula and the related literature.

Table 2. Phytoconstituents identified in Artemisia annua sample through MS analysis.

\begin{tabular}{|c|c|c|c|c|c|}
\hline Compound Name & m/z Detected & Theoretic $\mathrm{m} / \mathbf{z}$ & Formula & $\begin{array}{c}\text { Tentative } \\
\text { Identification }\end{array}$ & References \\
\hline 1 & 76.09 & 76.07 & $\mathrm{C}_{2} \mathrm{H}_{5} \mathrm{NO}_{2}+$ & Glycine & {$[32,61]$} \\
\hline 2 & 90.11 & 90.097 & $\mathrm{C}_{3} \mathrm{H}_{7} \mathrm{O}_{2}+$ & Alanine & {$[32,61]$} \\
\hline 3 & 103.19 & 103.17 & $\mathrm{C}_{6} \mathrm{H}_{14} \mathrm{O}+$ & Hexanol & {$[7,8,10,18,23,28,33,36,37,62]$} \\
\hline 4 & 106.12 & 106.09 & $\mathrm{C}_{3} \mathrm{H}_{7} \mathrm{NO}_{3}+$ & Serine & {$[32,61]$} \\
\hline 5 & 113.10 & 113.09 & $\mathrm{C}_{4} \mathrm{H}_{4} \mathrm{~N}_{2} \mathrm{O}_{2}+$ & Uracil & {$[7,8,10,18,24,30,35,38,39,61]$} \\
\hline 6 & 118.13 & 118.14 & $\mathrm{C}_{5} \mathrm{H}_{11} \mathrm{NO}_{2}+$ & Valine & {$[32,61]$} \\
\hline 7 & 120.05 & 120.03 & $\mathrm{C}_{4} \mathrm{H}_{9} \mathrm{NO}_{3}+$ & Threonine & {$[32,61]$} \\
\hline 8 & 129.23 & 129.21 & $\mathrm{C}_{8} \mathrm{H}_{16} \mathrm{O}+$ & Caprylaldehyde & {$[7,8,18,23,28,32,36,37,62]$} \\
\hline 9 & 132.14 & 132.13 & $\mathrm{C}_{5} \mathrm{H}_{9} \mathrm{NO}_{3}+$ & L-hydroxyproline & {$[32,61]$} \\
\hline 10 & 132.17 & 132.18 & $\mathrm{C}_{6} \mathrm{H}_{13} \mathrm{NO}_{2}+$ & Leucine & {$[32,61]$} \\
\hline 11 & 134.09 & 134.10 & $\mathrm{C}_{4} \mathrm{H}_{7} \mathrm{NO}_{4}+$ & Aspartic acid & {$[32,61]$} \\
\hline 12 & 135.23 & 135.22 & $\mathrm{C}_{10} \mathrm{H}_{14}{ }^{+}$ & p-Cymene & [63] \\
\hline 13 & 136.21 & 136.19 & $\mathrm{C}_{7} \mathrm{H}_{5} \mathrm{NS}+$ & Benzothiazole & {$[7,8]$} \\
\hline 14 & 137.25 & 137.23 & $\mathrm{C}_{10} \mathrm{H}_{16}+$ & Limonene & $\begin{array}{c}{[9-12,14,19,20,22,23,46,52,59,63-} \\
65]\end{array}$ \\
\hline 15 & 139.15 & 139.12 & $\mathrm{C}_{7} \mathrm{H}_{6} \mathrm{O}_{3}+$ & Salicylic acid & {$[7-9,18,23,28,33,36,37,62]$} \\
\hline 16 & 147.16 & 147.14 & $\mathrm{C}_{9} \mathrm{H}_{6} \mathrm{O}_{2}+$ & Coumarin & $\begin{array}{c}{[9-12,14,19,20,22,23,46,52,59,60,} \\
63,64]\end{array}$ \\
\hline 17 & 147.20 & 147.19 & $\mathrm{C}_{6} \mathrm{H}_{14} \mathrm{~N}_{2} \mathrm{O}_{2+}$ & Lysine & {$[32,61]$} \\
\hline 18 & 148.12 & 148.13 & $\mathrm{C}_{5} \mathrm{H}_{9} \mathrm{NO}_{4}+$ & Glutamic acid & {$[32,61]$} \\
\hline 19 & 149.21 & 149.205 & $\mathrm{C}_{10} \mathrm{H}_{12} \mathrm{O}+$ & 4-Isopropylbenzaldehyde & {$[7,8,18,23,28,33,36,37]$} \\
\hline
\end{tabular}


Table 2. Cont.

\begin{tabular}{|c|c|c|c|c|c|}
\hline Compound Name & m/z Detected & Theoretic $\mathrm{m} / \mathrm{z}$ & Formula & $\begin{array}{c}\text { Tentative } \\
\text { Identification }\end{array}$ & References \\
\hline 20 & 150.22 & 150.20 & $\mathrm{C}_{6} \mathrm{H}_{11} \mathrm{NO}_{2} \mathrm{~S}+$ & Methionine & {$[32,61]$} \\
\hline 21 & 151.19 & 151.22 & $\mathrm{C}_{10} \mathrm{H}_{14} \mathrm{O}+$ & Cuminol & {$[7,8,18,23,28,33,36,37,62]$} \\
\hline 22 & 153.25 & 153.233 & $\mathrm{C}_{10} \mathrm{H}_{16}+$ & Artemisia ketone & $\begin{array}{c}{[5-7,9,11-14,16,19-23,35,46,58-} \\
60,63,65]\end{array}$ \\
\hline 23 & 155.23 & 155.21 & $\mathrm{C}_{12} \mathrm{H}_{10}+$ & Capillene & {$[7,8,18,23,28,33,36,37,62]$} \\
\hline 24 & 155.27 & 155.25 & $\mathrm{C}_{10} \mathrm{H}_{18} \mathrm{O}+$ & Geraniol & $\begin{array}{c}{[9-12,14,19,20,22,23,46,52,59,61,} \\
63,64]\end{array}$ \\
\hline 25 & 156.17 & 156.16 & $\mathrm{C}_{6} \mathrm{H}_{9} \mathrm{~N}_{3} \mathrm{O}_{2}+$ & Histidine & {$[32,61]$} \\
\hline 26 & 157.29 & 157.26 & $\mathrm{C}_{10} \mathrm{H}_{20} \mathrm{O}+$ & Menthol & $\begin{array}{c}{[9-12,14,19,20,22,23,46,52,59,63,} \\
64,66]\end{array}$ \\
\hline 27 & 162.15 & 162.13 & $\mathrm{C}_{9} \mathrm{H}_{5} \mathrm{O}_{3}+$ & 4-Hydroxycoumarin & {$[7,8,10,18,23,28,33,36,37,62]$} \\
\hline 28 & 165.19 & 165.16 & $\mathrm{C}_{9} \mathrm{H}_{8} \mathrm{O}_{3}+$ & $\begin{array}{l}\text { 4-Hydroxycinnamic } \\
\text { acid }\end{array}$ & {$[7,8,10,18,23,28,33,36,37,62]$} \\
\hline 29 & 165.18 & 165.20 & $\mathrm{C}_{10} \mathrm{H}_{12} \mathrm{O}_{2}+$ & Eugenol & $\begin{array}{c}{[9-12,14,19,20,22,23,46,52,59,63,} \\
64,66]\end{array}$ \\
\hline 30 & 166.22 & 166.19 & $\mathrm{C}_{9} \mathrm{H}_{11} \mathrm{NO}_{2}+$ & L-phenylalanine & {$[32,61]$} \\
\hline 31 & 171.29 & 171.33 & $\mathrm{C}_{12} \mathrm{H}_{26}+$ & Dodecane & {$[7,8,18,23,28,33,36,37,62]$} \\
\hline 32 & 175.19 & 175.20 & $\mathrm{C}_{6} \mathrm{H}_{14} \mathrm{~N}_{4} \mathrm{O}_{2}+$ & L-arginine & {$[32,61]$} \\
\hline 33 & 181.17 & 181.16 & $\mathrm{C}_{9} \mathrm{H}_{8} \mathrm{O}_{4}+$ & Caffeic acid & {$[7,8,18,23,28,33,36,37,62]$} \\
\hline 34 & 183.19 & 183.17 & $\mathrm{C}_{9} \mathrm{H}_{10} \mathrm{O}_{4}+$ & $\begin{array}{l}\text { 2,4-Dihydroxy-6 } \\
\text { methoxyacetophe- } \\
\text { none }\end{array}$ & {$[7,8,18,23,28,33,35,37]$} \\
\hline 35 & 193.15 & 193.17 & $\mathrm{C}_{10} \mathrm{H}_{8} \mathrm{O}_{4}+$ & Scopoletin & $\begin{array}{c}{[9-12,14,19,20,22,23,46,52,59,63,} \\
64,66]\end{array}$ \\
\hline 36 & 197.21 & 197.20 & $\mathrm{C}_{10} \mathrm{H}_{12} \mathrm{O}_{4}+$ & Xanthoxylin & {$[7,8,18,23,28,33,36,37,62]$} \\
\hline 37 & 197.30 & 197.29 & $\mathrm{C}_{12} \mathrm{H}_{20} \mathrm{O}_{2+}$ & Artemisyl acetate & $\begin{array}{c}{[5-7,9,11-14,16,19-22,35,46,58-} \\
60,63,65]\end{array}$ \\
\hline 38 & 207.21 & 207.19 & $\mathrm{C}_{11} \mathrm{H}_{10} \mathrm{O}_{4}+$ & Scoparone & $\begin{array}{c}{[9-12,14,19,20,22,23,46,52,58,63,} \\
64,66]\end{array}$ \\
\hline 39 & 205.33 & 205.35 & $\mathrm{C}_{15} \mathrm{H}_{24}+$ & Germacrene D & $\begin{array}{c}{[9-12,14,19,20,22,23,46,52,58,63,} \\
64,66]\end{array}$ \\
\hline 40 & 207.39 & 207.37 & $\mathrm{C}_{15} \mathrm{H}_{26}+$ & Cadinene & $\begin{array}{c}{[9-12,14,19,20,22,23,46,52,58,63,} \\
64,66]\end{array}$ \\
\hline 41 & 220.23 & 220.24 & $\mathrm{C}_{10} \mathrm{H}_{13} \mathrm{~N}_{5} \mathrm{O}+$ & Zeatin & {$[7,8,18,23,28,33,36,37,62]$} \\
\hline 42 & 221.36 & 221.35 & $\mathrm{C}_{15} \mathrm{H}_{24} \mathrm{O}+$ & Spathulenol & $\begin{array}{c}{[9-12,14,19,20,22,23,46,52,58,63,} \\
64,66]\end{array}$ \\
\hline 43 & 223.38 & 223.37 & $\mathrm{C}_{15} \mathrm{H}_{26} \mathrm{O}+$ & Farnesol & $\begin{array}{c}9-12,14,19,20,22,23,46,52,58,63, \\
64,66]\end{array}$ \\
\hline 44 & 229.35 & 229.37 & $\mathrm{C}_{14} \mathrm{H}_{28} \mathrm{O}_{2}+$ & Myristic acid & {$[7,8,18,23,28,33,36,37,62]$} \\
\hline 45 & 233.30 & 233.32 & $\mathrm{C}_{15} \mathrm{H}_{20} \mathrm{O}_{2}+$ & Alantolactone & [64] \\
\hline 46 & 235.29 & 235.33 & $\mathrm{C}_{15} \mathrm{H}_{22} \mathrm{O}_{2}+$ & Artemisinic acid & $\begin{array}{c}{[5-7,9,11-14,16,19-22,35,46,58-} \\
60,63,65] \\
\end{array}$ \\
\hline 47 & 237.37 & 237.35 & $\mathrm{C}_{15} \mathrm{H}_{24} \mathrm{O}_{2}+$ & Corymbolone & $\begin{array}{c}{[9-12,14,19,20,22,23,46,52,59,63,} \\
64,66]\end{array}$ \\
\hline 48 & 246.33 & 246.32 & $\mathrm{C}_{15} \mathrm{H}_{19} \mathrm{NO}_{2}+$ & Rupestine & $\begin{array}{c}9-12,14,19,20,22,23,46,52,59,63, \\
64,66]\end{array}$ \\
\hline 49 & 265.31 & 265.32 & $\mathrm{C}_{15} \mathrm{H}_{20} \mathrm{O}_{4}+$ & Abscisic acid & $\begin{array}{c}{[5-7,9,11-14,16,19-22,35,46,58-} \\
60,63,65]\end{array}$ \\
\hline 50 & 241.48 & 241.5 & $\mathrm{C}_{17} \mathrm{H}_{36^{+}}$ & Heptadecane & {$[7,8,18,23,28,33,36,37,62]$} \\
\hline 51 & 247.29 & 247.30 & $\mathrm{C}_{15} \mathrm{H}_{18} \mathrm{O}_{3}+$ & $\alpha$-Santonin & [64] \\
\hline
\end{tabular}


Table 2. Cont.

\begin{tabular}{|c|c|c|c|c|c|}
\hline Compound Name & m/z Detected & Theoretic $\mathbf{m} / \mathbf{z}$ & Formula & $\begin{array}{c}\text { Tentative } \\
\text { Identification }\end{array}$ & References \\
\hline 52 & 249.30 & 249.32 & $\mathrm{C}_{15} \mathrm{H}_{20} \mathrm{O}_{3}+$ & Arteannuin B & $\begin{array}{c}5-7,9,11-14,16,19-22,35,46,58- \\
60,63,65]\end{array}$ \\
\hline 53 & 255.35 & 255.5 & $\mathrm{C}_{18} \mathrm{H}_{38}+$ & n-Octadecane & {$[7,8,18,23,28,33,36,37,62]$} \\
\hline 54 & 257.43 & 257.42 & $\mathrm{C}_{16} \mathrm{H}_{32} \mathrm{O}_{2}+$ & Palmitic acid & {$[7,8,18,23,28,33,36,37,62]$} \\
\hline 55 & 263.42 & 263.40 & $\mathrm{C}_{17} \mathrm{H}_{26} \mathrm{O}_{2}+$ & $\alpha$-Bergamotol acetate & {$[7,8,18,23,28,33,36,37,62]$} \\
\hline 56 & 267.30 & 267.33 & $\mathrm{C}_{15} \mathrm{H}_{22} \mathrm{O}_{4}+$ & Germacranolide & [64] \\
\hline 57 & 271.39 & 271.40 & $\mathrm{C}_{15} \mathrm{H}_{10} \mathrm{O}_{5}+$ & Apigenin & {$[56-60,67-71]$} \\
\hline 58 & 273.27 & 273.25 & $\mathrm{C}_{15} \mathrm{H}_{12} \mathrm{O}_{5}+$ & Naringenin & {$[56-60,67-71]$} \\
\hline 59 & 281.34 & 281.32 & $\mathrm{C}_{15} \mathrm{H}_{20} \mathrm{O}_{5}+$ & Artemisitene & $\begin{array}{c}{[5-7,9,11-14,16,19-22,35,46,58-} \\
60,63,65]\end{array}$ \\
\hline 60 & 283.34 & 283.33 & $\mathrm{C}_{15} \mathrm{H}_{22} \mathrm{O}_{5}+$ & Artemisinine & $\begin{array}{c}5-7,9,11-14,16,19-22,35,46,58- \\
60,63,65]\end{array}$ \\
\hline 61 & 285.27 & 285.26 & $\mathrm{C}_{16} \mathrm{H}_{12} \mathrm{O}_{5}+$ & Acacetin & {$[56-60,67-71]$} \\
\hline 62 & 287.26 & 287.24 & $\mathrm{C}_{15} \mathrm{H}_{10} \mathrm{O}_{6}+$ & Luteolin & {$[56-60,67-71]$} \\
\hline 63 & 289.27 & 289.25 & $\mathrm{C}_{15} \mathrm{H}_{12} \mathrm{O}_{6}+$ & Eriodyctiol & {$[56-60,67-71]$} \\
\hline 64 & 297.51 & 297.50 & $\mathrm{C}_{20} \mathrm{H}_{40} \mathrm{O}+$ & Phytol & $\begin{array}{c}{[9-12,14,19,20,22,23,46,52,59,63,} \\
64,66]\end{array}$ \\
\hline 65 & 301.28 & 301.26 & $\mathrm{C}_{16} \mathrm{H}_{12} \mathrm{O}_{6}+$ & Rhamnocitrin & {$[56-60,67-71]$} \\
\hline 66 & 303.21 & 303.23 & $\mathrm{C}_{15} \mathrm{H}_{10} \mathrm{O}_{7+}$ & Quercetin & {$[56-60,68,69]$} \\
\hline 67 & 305.22 & 305.25 & $\mathrm{C}_{15} \mathrm{H}_{12} \mathrm{O}_{7+}$ & Taxifolin & {$[56-60,64,67-71]$} \\
\hline 68 & 315.31 & 315.29 & $\mathrm{C}_{17} \mathrm{H}_{14} \mathrm{O}_{6}+$ & Cirsimaritin & {$[56-60,64,67-71]$} \\
\hline 69 & 317.24 & 317.26 & $\mathrm{C}_{16} \mathrm{H}_{12} \mathrm{O}_{7+}$ & Rhamnetin & {$[56-60,67-71]$} \\
\hline 70 & 317.37 & 317.40 & $\mathrm{C}_{19} \mathrm{H}_{24} \mathrm{O}_{4}+$ & Capillartemisin B & {$[56-60,64]$} \\
\hline 71 & 319.20 & 319.23 & $\mathrm{C}_{15} \mathrm{H}_{10} \mathrm{O}_{8}+$ & Quercetagetin & {$[56-60,64,67-71]$} \\
\hline 72 & 324.57 & 324.60 & $\mathrm{C}_{22} \mathrm{H}_{44} \mathrm{O}+$ & 2-Docosanone & {$[7,8,18,23,28,33,36,37,62]$} \\
\hline 73 & 331.26 & 331.29 & $\mathrm{C}_{17} \mathrm{H}_{14} \mathrm{O}_{7+}$ & Rhamnazin & {$[56-60,64,67-71]$} \\
\hline 74 & 333.24 & 333.26 & $\mathrm{C}_{16} \mathrm{H}_{12} \mathrm{O}_{8}+$ & Laricitrin & {$[56-60,64,67-71]$} \\
\hline 75 & 339.67 & 339.70 & $\mathrm{C}_{24} \mathrm{H}_{50}+$ & n-Tetracosane & {$[7,8,18,23,28,33,36,37,62]$} \\
\hline 76 & 345.32 & 345.30 & $\mathrm{C}_{18} \mathrm{H}_{16} \mathrm{O}_{7+}$ & Eupatorine & {$[56-60,64,67-71]$} \\
\hline 77 & 347.27 & 347.30 & $\mathrm{C}_{17} \mathrm{H}_{14} \mathrm{O}_{8}+$ & Syringetin & {$[56-60,64,67-71]$} \\
\hline 78 & 353.68 & 353.70 & $\mathrm{C}_{25} \mathrm{H}_{52}+$ & n-Pentacosane & {$[7,8,18,23,28,33,36,37,62]$} \\
\hline 79 & 354.29 & 354.31 & $\mathrm{C}_{16} \mathrm{H}_{18} \mathrm{O}_{9}+$ & Scopoline & $\begin{array}{c}{[9-12,14,19,20,22,23,46,52,59,63,} \\
64,66]\end{array}$ \\
\hline 80 & 359.31 & 359.30 & $\mathrm{C}_{19} \mathrm{H}_{18} \mathrm{O}_{7+}$ & Retusin & {$[56-60,64,67-71]$} \\
\hline 81 & 361.33 & 361.30 & $\mathrm{C}_{18} \mathrm{H}_{16} \mathrm{O}_{8}+$ & Chrysosplenol D & {$[56-60,64,67-71]$} \\
\hline 82 & 367.71 & 367.7 & $\mathrm{C}_{26} \mathrm{H}_{54}+$ & n-Hexacosane & {$[7,8,18,23,28,33,36,37,62]$} \\
\hline 83 & 375.28 & 375.30 & $\mathrm{C}_{19} \mathrm{H}_{18} \mathrm{O}_{8}+$ & Chrysosplenetin & {$[56-60,64,67-71]$} \\
\hline 84 & 389.39 & 389.40 & $\mathrm{C}_{20} \mathrm{H}_{20} \mathrm{O}_{8}+$ & Artemitin & {$[9,16,17,21,23,56-60,65]$} \\
\hline 85 & 375.28 & 375.30 & $\mathrm{C}_{19} \mathrm{H}_{18} \mathrm{O}_{8}+$ & Casticin & {$[56-60,64,67-71]$} \\
\hline 86 & 377.41 & 377.40 & $\mathrm{C}_{20} \mathrm{H}_{24} \mathrm{O}_{7+}$ & Euparotin & {$[56-60]$} \\
\hline 87 & 411.68 & 411.70 & $\mathrm{C}_{30} \mathrm{H}_{50}+$ & Squalene & $\begin{array}{c}{[9-12,14,19,20,22,23,46,52,59,63,} \\
64,66]\end{array}$ \\
\hline 88 & 413.72 & 413.70 & $\mathrm{C}_{29} \mathrm{H}_{48} \mathrm{O}+$ & Stigmasterol & {$[7,8,18,24,30,35,38,39]$} \\
\hline 89 & 415.67 & 415.70 & $\mathrm{C}_{29} \mathrm{H}_{50} \mathrm{O}+$ & $\beta$-Sitosterol & {$[7,8,18,23,28,33,36,37,62]$} \\
\hline 90 & 425.69 & 425.70 & $\mathrm{C}_{30} \mathrm{H}_{48} \mathrm{O}+$ & Taraxasterone & $\begin{array}{c}9-12,14,19,20,22,23,46,52,59,63, \\
64,66]\end{array}$ \\
\hline 91 & 427.71 & 427.70 & $\mathrm{C}_{30} \mathrm{H}_{50} \mathrm{O}+$ & Beta-amyrin & $\begin{array}{c}{[9-12,14,19,20,22,23,46,52,59,63,} \\
64,66]\end{array}$ \\
\hline
\end{tabular}


Table 2. Cont.

\begin{tabular}{|c|c|c|c|c|c|}
\hline Compound Name & m/z Detected & Theoretic $\mathrm{m} / \mathrm{z}$ & Formula & $\begin{array}{c}\text { Tentative } \\
\text { Identification }\end{array}$ & References \\
\hline 92 & 433.37 & 433.40 & $\mathrm{C}_{21} \mathrm{H}_{20} \mathrm{O}_{10^{+}}$ & $\begin{array}{c}\text { Apigenin } \\
\text { 7-O-glucoside }\end{array}$ & [64] \\
\hline 94 & 443.52 & 443.50 & $\mathrm{C}_{25} \mathrm{H}_{30} \mathrm{O}_{7}+$ & Tomentin A & $\begin{array}{c}{[9-12,14,19,20,22,23,46,52,59,63,} \\
64,66]\end{array}$ \\
\hline 95 & 447.38 & 447.40 & $\mathrm{C}_{22} \mathrm{H}_{22} \mathrm{O}_{10^{+}}$ & $\begin{array}{l}\text { Kaempferide } \\
\text { 3-rhamnoside }\end{array}$ & [57] \\
\hline 96 & 449.37 & 449.40 & $\mathrm{C}_{21} \mathrm{H}_{20} \mathrm{O}_{11}+$ & Cymaroside & {$[56-60,64,67-71]$} \\
\hline 97 & 457.71 & 457.70 & $\mathrm{C}_{30} \mathrm{H}_{48} \mathrm{O}_{3}+$ & Oleanic acid & {$[7,8,18,22,27,31,34,35,60]$} \\
\hline 98 & 465.38 & 465.40 & $\mathrm{C}_{21} \mathrm{H}_{20} \mathrm{O}_{12}+$ & Isoquercetin & {$[56-60,64,67-71]$} \\
\hline 99 & 495.43 & 495.40 & $\mathrm{C}_{22} \mathrm{H}_{22} \mathrm{O}_{13}+$ & Patuletin 3-glucoside & [64] \\
\hline 100 & 505.37 & 505.40 & $\mathrm{C}_{18} \mathrm{H}_{32} \mathrm{O}_{16}+$ & Sophorotriose & [64] \\
\hline 101 & 517.42 & 517.40 & $\mathrm{C}_{25} \mathrm{H}_{24} \mathrm{O}_{12}+$ & Cynarine & {$[7,8,18,22,27,31,34,35,60]$} \\
\hline 102 & 577.77 & 577.80 & $\mathrm{C}_{35} \mathrm{H}_{60} \mathrm{O}_{6}+$ & Daucosterol & {$[7,8,18,22,27,31,34,35,60]$} \\
\hline 103 & 611.51 & 611.50 & $\mathrm{C}_{27} \mathrm{H}_{30} \mathrm{O}_{16}+$ & Rutin & {$[56-60,64,67-72]$} \\
\hline
\end{tabular}

\subsection{Screening and Classification of the Differential Metabolites}

The 103 phytochemicals identified through mass spectroscopy were assigned to different chemical classes: terpenoids and sesquiterpenoids (27.2\%), flavonoids $(24.2 \%)$, amino acids $(12.6 \%)$, hydrocarbons $(6.8 \%)$, coumarins $(4.85 \%)$, phenolic acids $(2.9 \%)$, sterol and steroids $(2.9 \%)$, fatty acids $(2.9 \%)$, glycosides $(1.9 \%)$, hydrocarbons $(6.8 \%)$, organic acids and esters $(3.8 \%)$, carbohydrates $(0.97 \%)$ and miscellaneous (Table 3$)$. Terpenoids and sesquiterpenoids, flavonoids and amino acids constitute the largest group of bioactive compounds from Artemisia annua. The distribution of identified metabolites in various chemical categories is listed in Table 3.

Table 3. Classification of metabolites from Artemisia annua sample on various chemical categories.

\begin{tabular}{|c|c|}
\hline Chemical Class & Metabolite Name \\
\hline \multirow{14}{*}{ Amino Acids } & Glycine \\
\hline & Alanine \\
\hline & Serine \\
\hline & Valine \\
\hline & Threonine \\
\hline & L-hydroxyproline \\
\hline & Leucine \\
\hline & Aspartic acid \\
\hline & Lysine \\
\hline & Glutamic acid \\
\hline & Methionine \\
\hline & Hystidine \\
\hline & L-phenylalanine \\
\hline & L-arginine \\
\hline
\end{tabular}


Table 3. Cont.

\begin{tabular}{|c|c|}
\hline Chemical Class & Metabolite Name \\
\hline \multirow{28}{*}{ Terpenoids and Sesquiterpenoids } & Artemisine \\
\hline & Artemisinine \\
\hline & Limonele \\
\hline & p-Cymene \\
\hline & Beta-amyrin \\
\hline & Artesimic acid \\
\hline & Eugenol \\
\hline & Menthol \\
\hline & Artemisia ketone \\
\hline & Spathulenol \\
\hline & Artemisyl acetate \\
\hline & Artemisinic acid \\
\hline & Phytol \\
\hline & Rupestine \\
\hline & $\alpha$-Santonin \\
\hline & Arteannuin B \\
\hline & Farnesol \\
\hline & Corymbolone \\
\hline & Abscisic acid \\
\hline & Alantolactone \\
\hline & Artemisitene \\
\hline & Geraniol \\
\hline & Squalene \\
\hline & Taraxasterone \\
\hline & Beta-amyrin \\
\hline & Germacranolide \\
\hline & Germacrene D \\
\hline & Cadinene \\
\hline \multirow{5}{*}{ Coumarins } & Scopoletin \\
\hline & Tomentin A \\
\hline & Coumarin \\
\hline & Scopolin \\
\hline & Scoparone \\
\hline
\end{tabular}


Table 3. Cont.

\begin{tabular}{|c|c|}
\hline Chemical Class & Metabolite Name \\
\hline \multirow{25}{*}{ Flavonoids } & Apigenin \\
\hline & Chrysosplenetin \\
\hline & Rhamnazin \\
\hline & Luteolin \\
\hline & Naringenin \\
\hline & Capillartemisin B \\
\hline & Rutin \\
\hline & Quercetin \\
\hline & Quercetagetin \\
\hline & Acacetin \\
\hline & Rhamnetin \\
\hline & Eupatorin \\
\hline & Syringetin \\
\hline & Laricitrin \\
\hline & Eriodictiol \\
\hline & Casticin \\
\hline & Chrysosplenol D \\
\hline & Retusin \\
\hline & Cynaroside \\
\hline & Artemitin \\
\hline & Taxifolin \\
\hline & Isoquercetin \\
\hline & Rhamnocitrin \\
\hline & Kaempferide 3-rhamnoside \\
\hline & Cirsimaritin \\
\hline \multirow{3}{*}{ Phenolic Acids } & 4-Hydroxycoumarin \\
\hline & 4-Hydroxycinnamic acid \\
\hline & Caffeic acid \\
\hline \multirow{3}{*}{ Sterol and Steroids } & $\beta$-Sitosterol \\
\hline & Stigmasterol \\
\hline & Daucosterol \\
\hline \multirow{3}{*}{ Fatty Acid } & Oleanic acid \\
\hline & Palmitic acid \\
\hline & Myristic acid \\
\hline \multirow{7}{*}{ Hydrocarbons } & n-Octadecane \\
\hline & Heptadecane \\
\hline & n-Tetracosane \\
\hline & n-Hexacosane \\
\hline & Dodecane \\
\hline & $\mathrm{n}$-Pentacosane \\
\hline & Capillene \\
\hline \multirow{2}{*}{ Glycoside } & Patuletin 3-glucoside \\
\hline & Apigenin 7-O-glucoside \\
\hline Carbohydrates & Sophorotriose \\
\hline \multirow{5}{*}{ Aldehyde and Ketone } & Caprylaldehyde \\
\hline & 4-Isopropylbenzaldehyde \\
\hline & Hexanol \\
\hline & 2-Docosanone \\
\hline & 2,4-Dihydroxy-6-methoxyacetophenone \\
\hline \multirow{4}{*}{ Organic Acids and Esters } & Salicylic acid \\
\hline & $\alpha$-Bergamotol acetate \\
\hline & Xanthoxylin \\
\hline & Cynarine \\
\hline
\end{tabular}


Table 3. Cont.

\begin{tabular}{|c|c|}
\hline Chemical Class & Metabolite Name \\
\hline \multirow{4}{*}{ Other } & Uracil \\
\hline & Cuminol \\
\hline & Benzothiazole \\
\hline & Zeatin \\
\hline
\end{tabular}

On the basis of the data analysis reported in Table 3, the metabolites classification chart was obtained, represented in Figure 3.

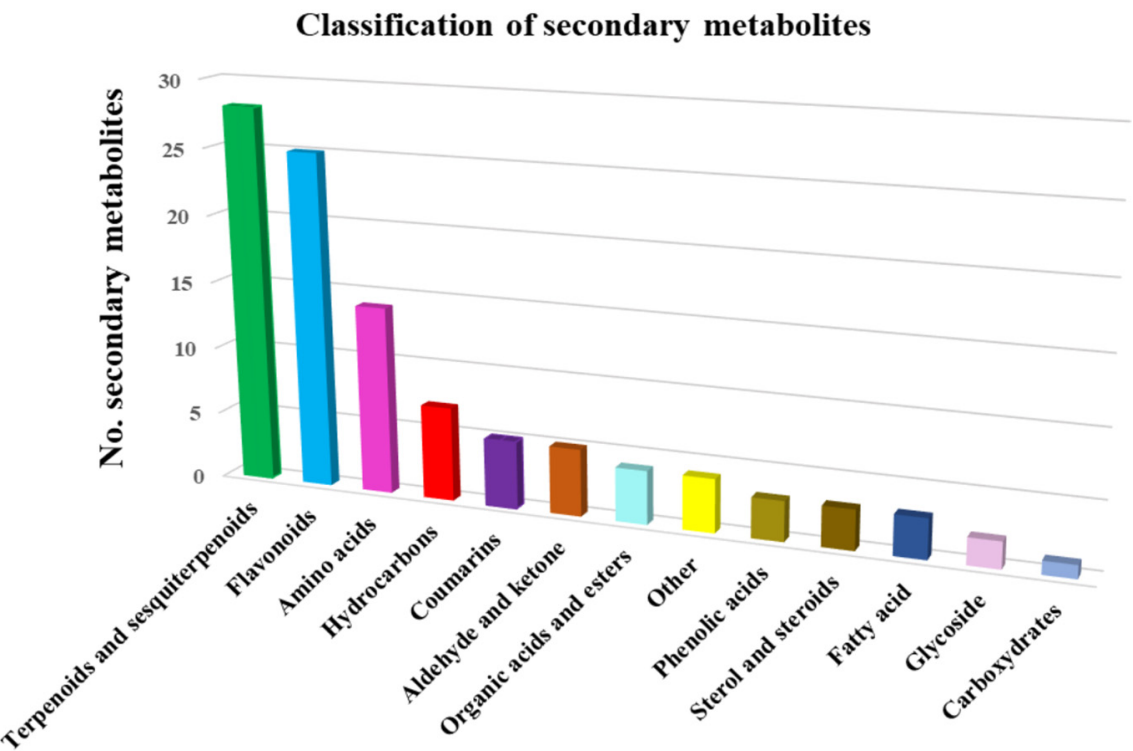

Figure 3. Artemisia annua metabolite classification.

Amino acids a total of 14 different amino acids were identified in the plant extract. Additionally, the essential amino acids (valine, leucine, methionine, hystidine and Lphenylalanine) represent $35 \%$ of them. The non-essential amino acids are present in a much larger proportion (65\%), being representatives of: glycine, alanine, serine, arginine, threonine, acid aspartic, lysine and glutamic acid [31-33,73]. As was reported, amino acids exhibit antitumoral, antiproliferative and immunomodulant activity [31-33,73].

Terpenoids found in the Artemisia annua sample are the one of the major constituents of the total identified metabolites. Previous studies on the therapeutic effect of terpenoids have demonstrated their antimicrobial, antibacterial, antifungal, analgesic and anti-insect activity [74].

Sesquiterpenes, another important class of metabolites from Artemisia annua, were shown to have antitumoral, antiplasmodial, anti-inflammatory and anti-allergic properties. Sesquiterpenes lactones isolated from Artemisia annua are used in antimalaria drugs [75,76].

Coumarins are metabolites highly relevant to human health. Recent studies on coumarins isolated from plants have shown antioxidant, antimicrobial, antiviral, antifungal, and antiparasitic, anti-diabetic, analgesic, anti-neurodegenerative, and anti-inflammatory activity. Moreover, coumarins have been demonstrated to stimulate the immunologic response and are used in the therapy of different tumors: leukemia, renal and prostate tumors, melanoma and breast cancer [77,78].

Flavonoids were another major category of metabolites identified in the plant sample. A total of 25 different flavonoids were found in the Artemisia annua sample. These compounds exhibit antioxidant, antitumoral, anti-inflammatory, antimicrobial, anti-cholinesterase, neurodegenerative disease (Alzheimer) and atherosclerosis prevention effects [9-81]. 
Phenolic acids have shown anti-inflammatory, antioxidant, antimicrobial, neuroprotective, antidiabetic and anticancer effects $[82,83]$.

Sterol and steroids from herbs act as antitumoral, anti-inflammatory, antioxidant antiatherosclerotic agents [84].

Fatty acids are involved in neuroprotection and cardiovascular protection mechanisms. Recent studies reported their beneficial role in autoimmune and neurodegenerative diseases, including Alzheimer disease (AD) [85].

Carbohydrates have shown anti-inflammatory, antioxidant, antiviral, antibacterial, antidiabetic, antitumoral, immunomodulatory and cardioprotective activity [86-88].

Glycosides from herbs showed antitumoral activity, mainly on leukemia and gastric cancer [89].

\subsection{Nano-Carrier System Based on Magnetic Nanoparticles of $\mathrm{Fe}_{3} \mathrm{O}_{4}$}

The development of an efficient and selective drug nano-carrier system required an optimal ratio between the herb and magnetic nanoparticles in order to provide the highest biological activity and functionality (selectivity and vectorization). Recent studies regarding types of nano-drug systems have reported the specific bioactive phytochemicals that were loaded into the magnetic nanoparticles $[90,91]$.

\subsection{FT-IR Spectroscopy}

The incorporation of herb phytochemicals into the pores of $\mathrm{Fe}_{3} \mathrm{O}_{4}$ nanoparticles was successfully achieved and was confirmed through FT-IR spectroscopy. Figure 4 presents the spectra of the herb, $\mathrm{Fe}_{3} \mathrm{O}_{4}$ nanoparticles and the nano-carrier system.

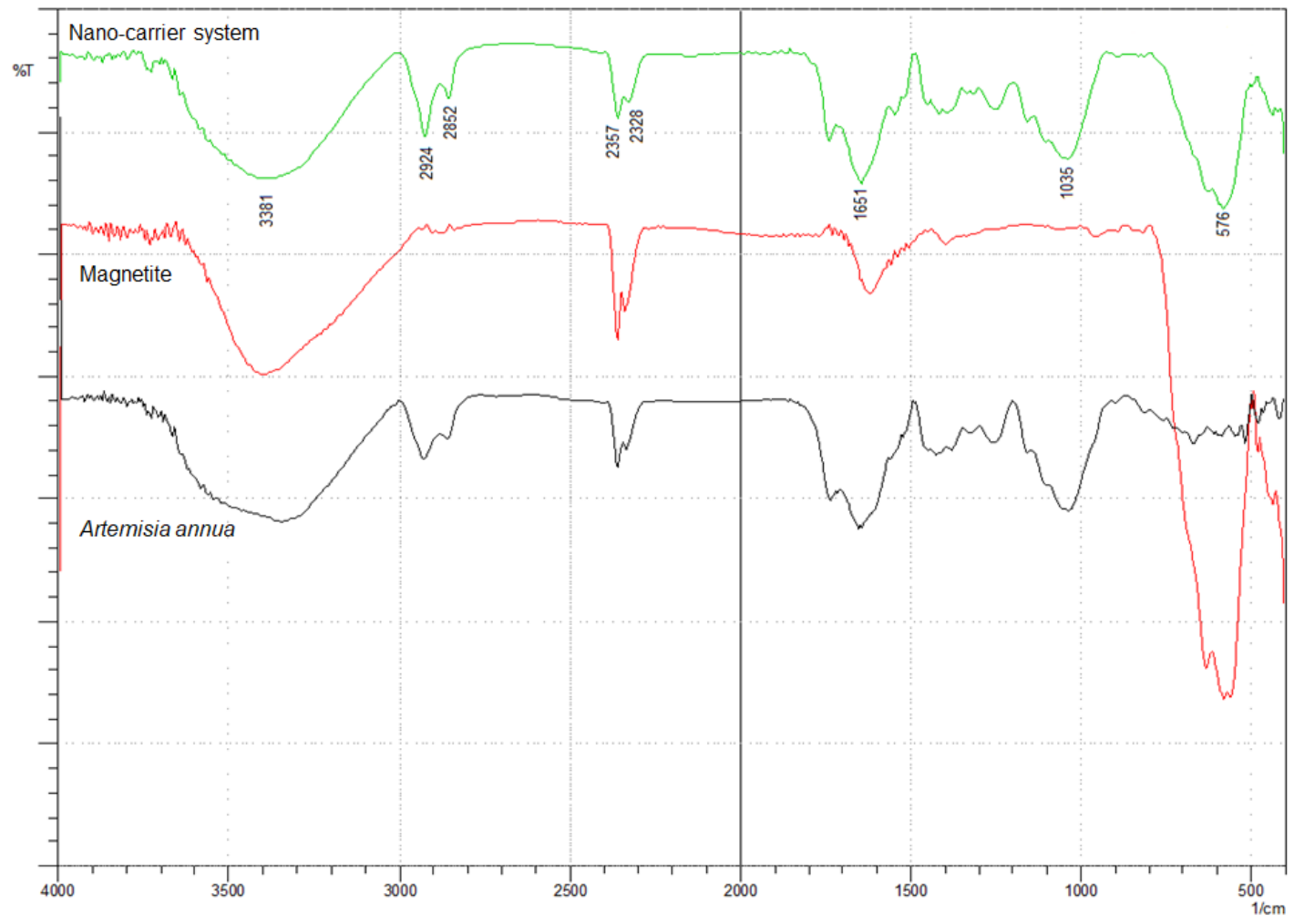

Figure 4. FT-IR spectra of Artemisia annua, magnetite nanoparticles and nano-carrier system.

The FT-IR spectra of the herb display the characteristic absorption peaks of Artemisia annua (Figure 4). The characteristic group frequencies of different organic molecules detected in Artemisia annua can be attributed to flavonoids (1703, 1580, 1460, 630 and $\left.575 \mathrm{~cm}^{-1}\right)$, amino acids $\left(1651,1580,1555\right.$ and $\left.1545 \mathrm{~cm}^{-1}\right)$, terpenoids (1740, 1651 and 
$\left.810 \mathrm{~cm}^{-1}\right)$, carbohydrates $\left(3381,1462,1126\right.$ and $\left.840 \mathrm{~cm}^{-1}\right)$ and fatty acids $(2925,2852,1250$ and $\left.720 \mathrm{~cm}^{-1}\right)[47,92-101]$.

Withal, the band at $1737 \mathrm{~cm}^{-1}$ is the characteristic absorption peak of Artemisia annua assigned to $\delta$-lactone group [47].

Two main broad metal-oxygen bands are seen in the IR spectra of $\mathrm{Fe}_{3} \mathrm{O}_{4}$ nanoparticles (Figure 4) in the range $400-600 \mathrm{~cm}^{-1}$. The highest vibration band at $576 \mathrm{~cm}^{-1}$ is assigned to the stretching vibrations of $\mathrm{M}_{\text {tetra }} \mathrm{O}$ bond in the tetrahedral voids, and the lowest band at $410 \mathrm{~cm}^{-1}$ (partially visible) corresponds to the stretching vibrations of the $\mathrm{M}_{\mathrm{octa}} \mathrm{O}$ bond in the octahedral void peak [102-104].

The spectra of the nano-carrier system (Figure 4) display the characteristic peaks of the herb as well as the metal-oxygen vibration bands at $576 \mathrm{~cm}^{-1}$ and at $410 \mathrm{~cm}^{-1}$, which confirm the incorporation of the herb into the pores of $\mathrm{Fe}_{3} \mathrm{O}_{4}$ nanoparticles [47].

\subsection{X-ray Diffraction Spectroscopy}

Figures 5-8 present the XRD patterns of $\mathrm{Fe}_{3} \mathrm{O}_{4}$ nanoparticles, the herb and the nanocarrier system.

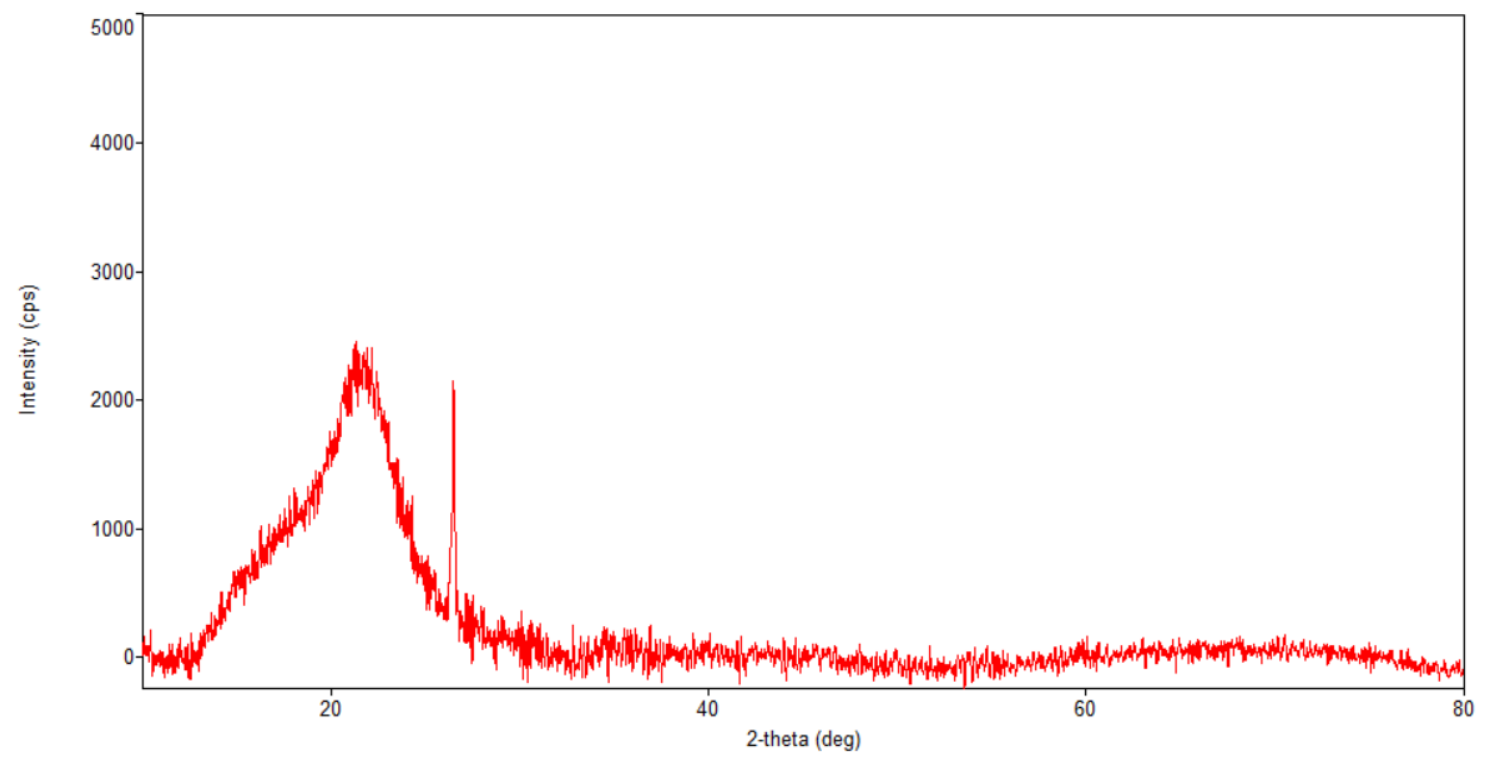

Figure 5. Powder XRD patterns of Artemisia annua.

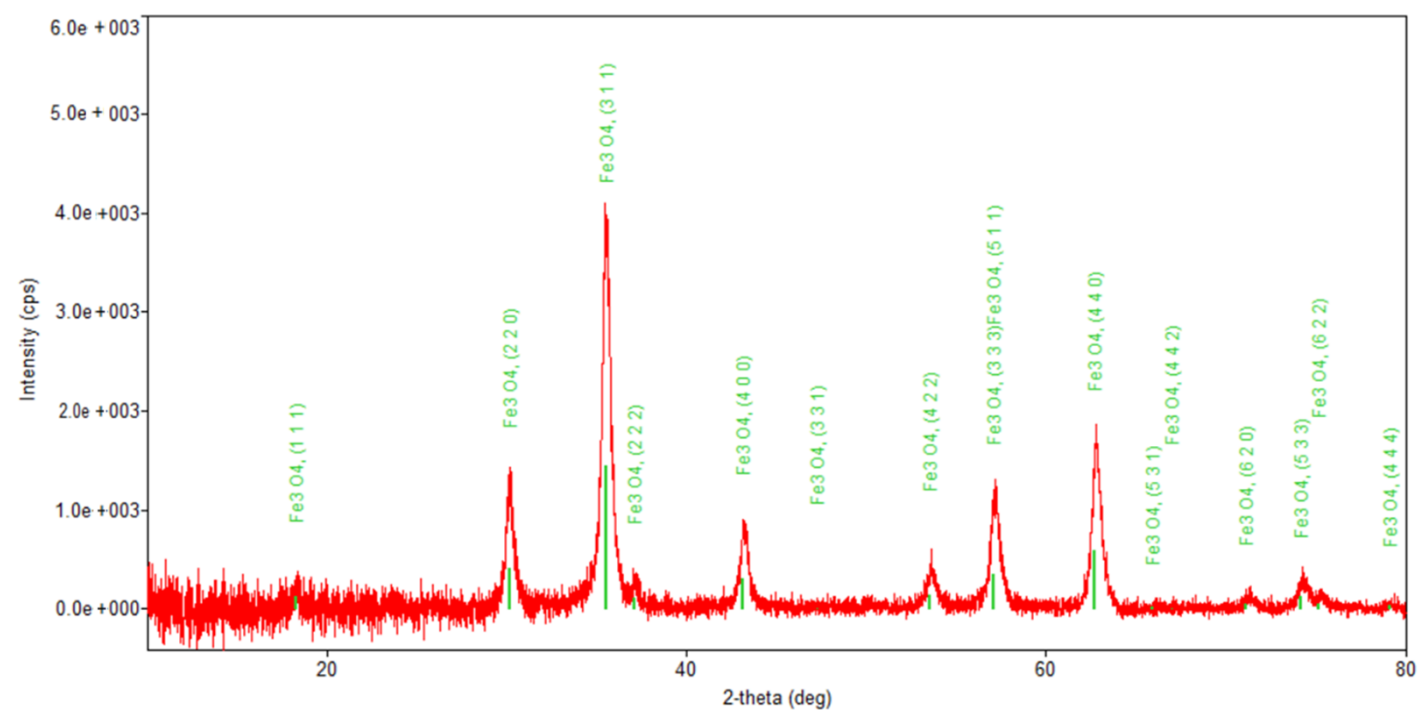

Figure 6. Powder XRD patterns of $\mathrm{Fe}_{3} \mathrm{O}_{4}$ nanoparticles. 


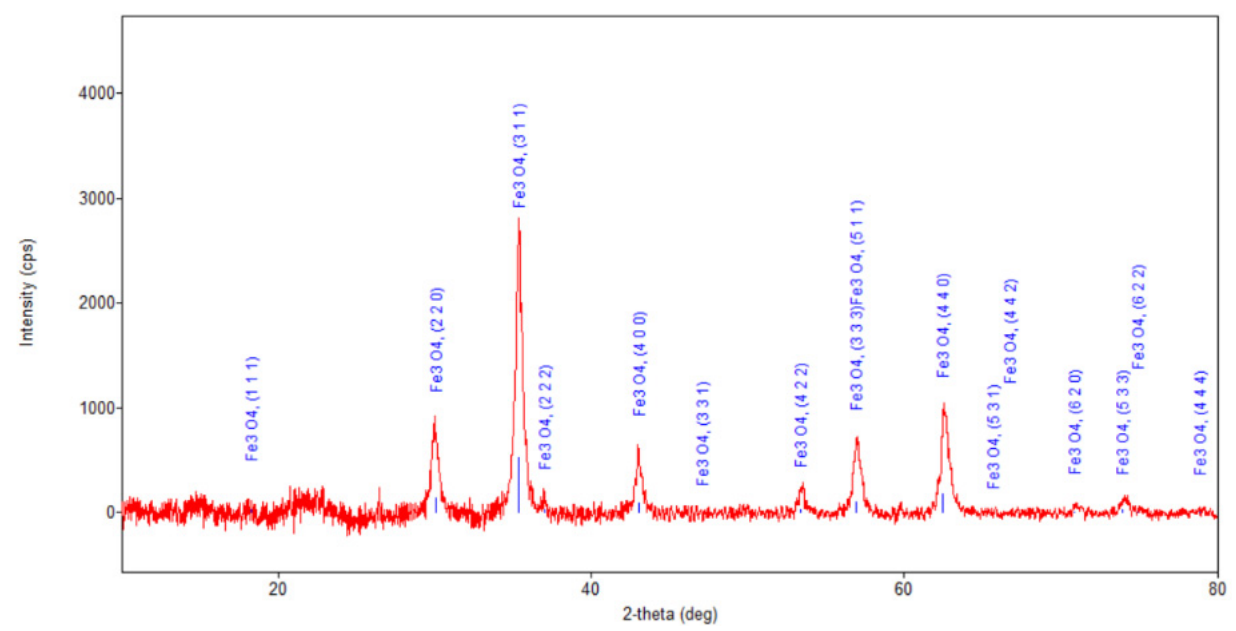

Figure 7. Powder XRD patterns of nano-carrier system.

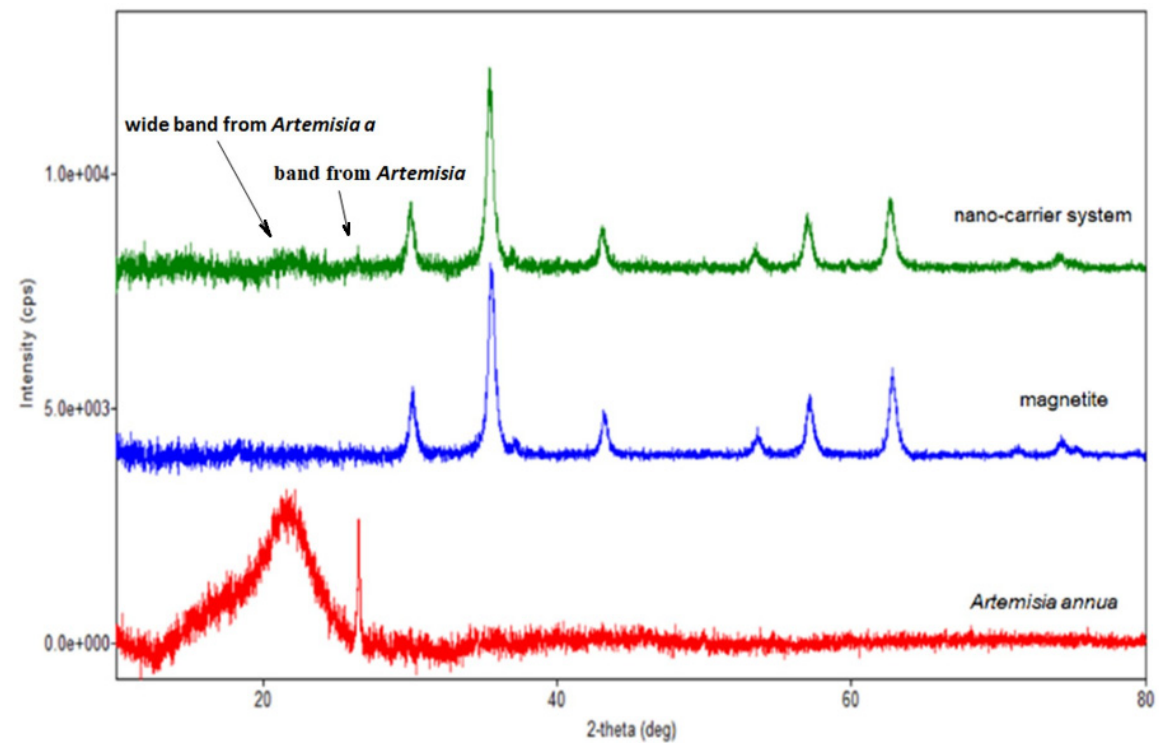

Figure 8. Overlap of the XRD patterns of herb, magnetite and nano-carrier system.

Regarding the diffraction pattern of the herb (Figure 5), in the range of $13-26^{\circ}$, a wide band that is characteristic of some amorphous phases can be observed. This wide band is also found attenuated in the diffraction pattern of the nano-carrier system (Figure 7 and Figure 8). Additionally, in the diffraction pattern of the $\mathrm{Fe}_{3} \mathrm{O}_{4}$ nanoparticles (Figure 6), only the peaks of the single crystalline spinel phase $\mathrm{Fe}_{3} \mathrm{O}_{4}$ (average crystallite size $10.9 \mathrm{~nm}$ ) are present.

In the diffraction pattern of the mixture (Figures 7 and 8), crystalline spinel phase $\mathrm{Fe}_{3} \mathrm{O}_{4}$ nanoparticles with an average crystallite size of $12.9 \mathrm{~nm}$ were identified. A peak at $\sim 26.5^{\circ}$ and a band between $13-26^{\circ}$ were also present but much attenuated in the spectrum of Artemisia annua.

\subsection{Scanning Electron Microscopy (SEM)}

The SEM micrographs of the herb, magnetic nanoparticles and the nano-carrier system are shown in Figures 9-11. 


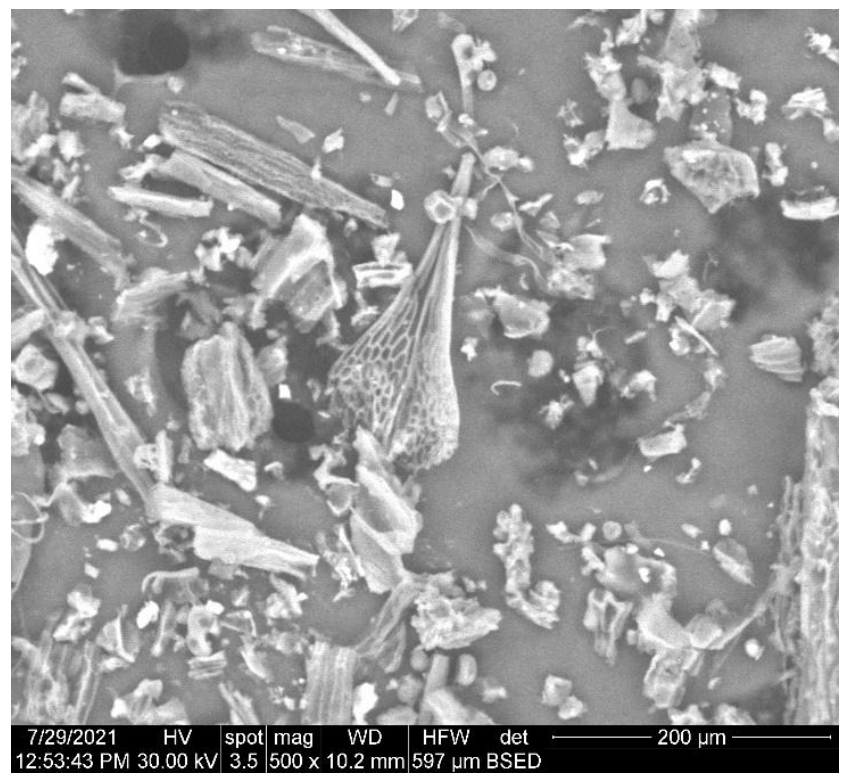

Figure 9. High-resolution SEM images of Artemisia annua.

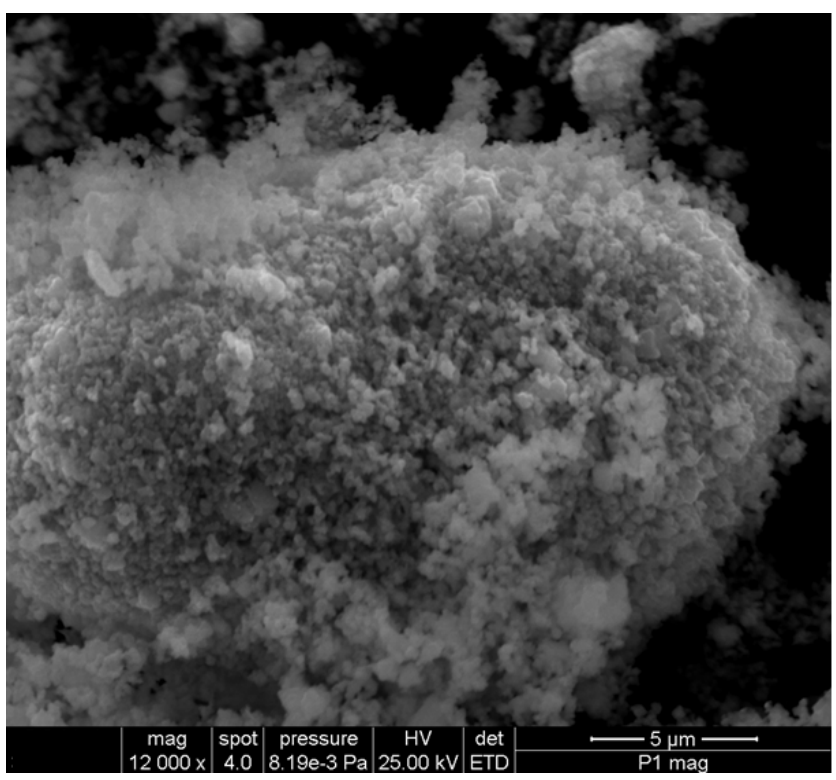

Figure 10. High-resolution SEM images of $\mathrm{Fe}_{3} \mathrm{O}_{4}$ nanoparticles.

As can be seen in the SEM image (Figure 9), the particles of Artemisia anuua shown are in the form of micron-sized fibers and irregular shape particles. The incorporation of herb phytochemicals into the pores of $\mathrm{Fe}_{3} \mathrm{O}_{4}$ nanoparticles was also confirmed via the scanning electron microscopy (SEM) images of $\mathrm{Fe}_{3} \mathrm{O}_{4}$ nanoparticles (Figure 10) and $\mathrm{Fe}_{3} \mathrm{O}_{4}$ loaded with the herb (Figure 11). The SEM image of $\mathrm{Fe}_{3} \mathrm{O}_{4}$ nanoparticles loaded with the herb (Figure 11b) shows a powder that consists of agglomerations of round nanoparticles with dimensions between 5 and $30 \mathrm{~nm}$, as well as irregular shapes with dimensions greater than $60 \mathrm{~nm}$. In the SEM image at high magnification (Figure 11a), irregular shape particles in $\mathrm{Fe}_{3} \mathrm{O}_{4}$ nanoparticles' surface modification with herbs can be seen. 


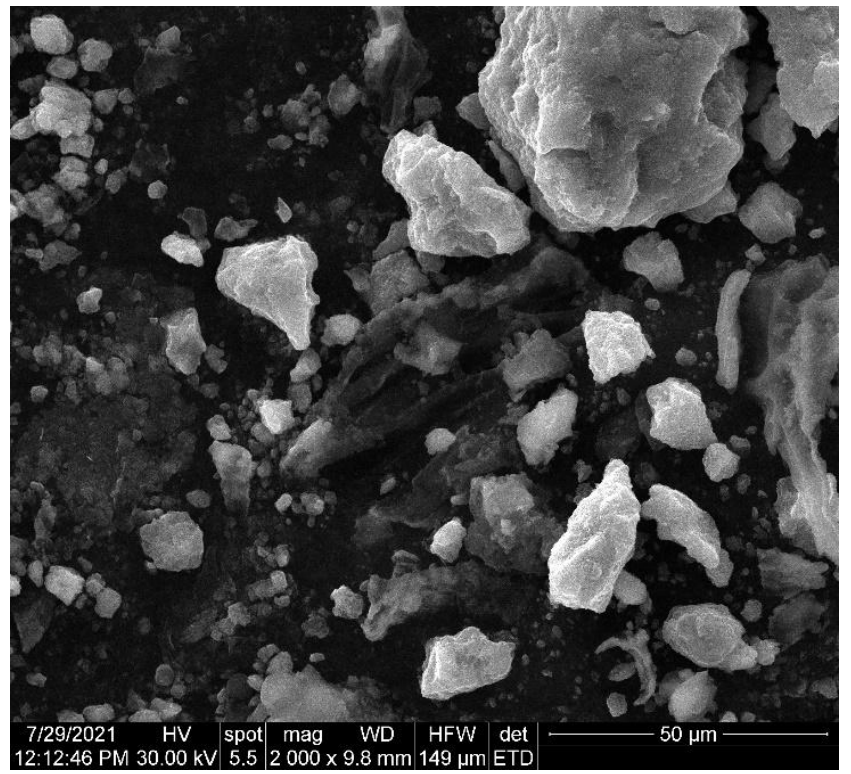

(a)

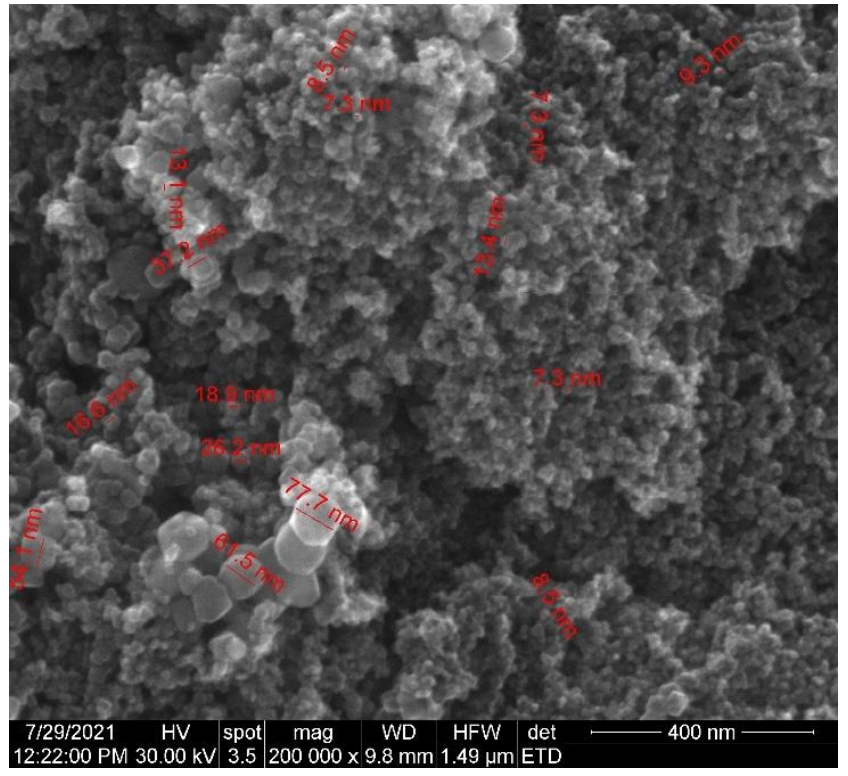

(b)

Figure 11. (a) $\mathrm{SEM}$ images of $\mathrm{Fe}_{3} \mathrm{O}_{4}$ nanoparticles loaded with herb at high magnification. (b) $\mathrm{SEM}$ images of $\mathrm{Fe}_{3} \mathrm{O}_{4}$ nanoparticles loaded with herb at low magnification.

\subsection{Magnetic Properties}

The magnetic properties of the $\mathrm{Fe}_{3} \mathrm{O}_{4}$ nanoparticles and the nano-carrier system were investigated at a low-frequency driving field $(50 \mathrm{~Hz})$ by means of an induction hysteresis graph [105]. It was found that both samples exhibit ferromagnetic behavior with narrow hysteresis loops (see Figures 12 and 13).

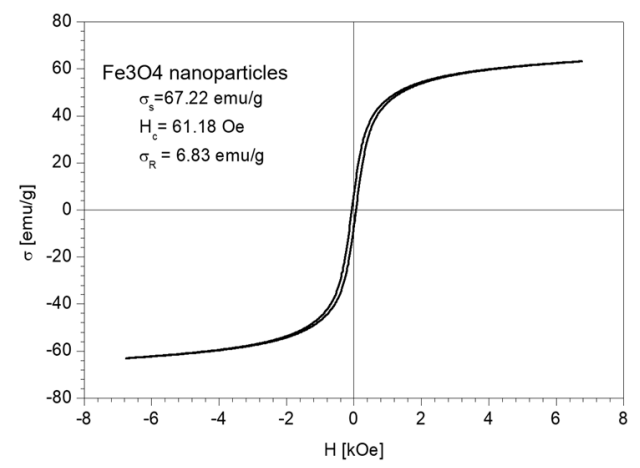

Figure 12. The hysteresis loop of $\mathrm{Fe}_{3} \mathrm{O}_{4}$ nanoparticles sample.

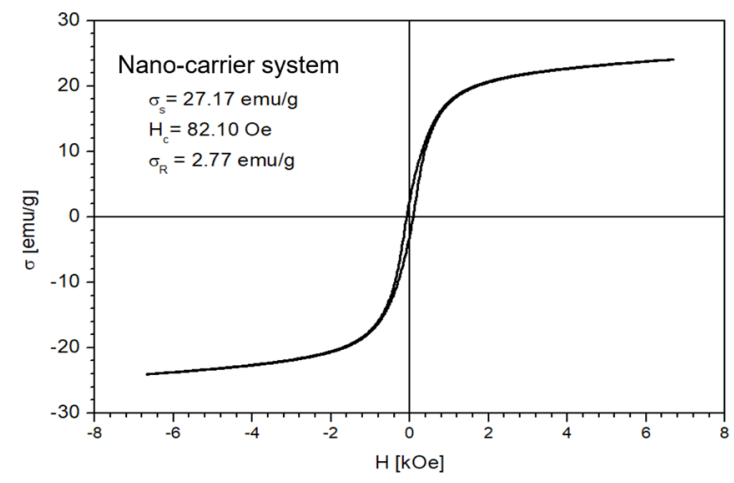

Figure 13. The hysteresis loop of nano-carrier system. 
From the measured hysteresis loops, the saturation magnetization $\left(\sigma_{\mathrm{S}}\right)$, the coercive field $\left(H_{\mathcal{C}}\right)$ and the remanent magnetization $\left(\sigma_{R}\right)$ were determined. The results are presented in Table 4.

Table 4. Magnetic properties of $\mathrm{Fe}_{3} \mathrm{O}_{4}$ nanoparticles and nano-carrier system.

\begin{tabular}{cccc}
\hline Sample & $\sigma_{\mathbf{S}}(\mathbf{e m u} / \mathbf{g})$ & $\mathbf{H}_{\mathbf{C}}(\mathbf{O e})$ & $\sigma_{\mathbf{R}}(\mathbf{e m u} / \mathbf{g})$ \\
\hline $\mathrm{Fe}_{3} \mathrm{O}_{4}$ nanoparticles & 67.22 & 61.18 & 6.83 \\
\hline Nano-carrier system & 27.17 & 82.10 & 2.77 \\
\hline
\end{tabular}

As expected, the saturation magnetization of the sample $\mathrm{Fe}_{3} \mathrm{O}_{4}$ nanoparticles is larger than that of nano-carrier system. Both samples have small values regarding the remnant ratio $\sigma_{R} / \sigma_{S}$ (in order of 0.1 ), which is an indication of the ease with which the magnetization reorients to the nearest easy axis magnetization direction after the removal of the magnetic field. The frequency dependence on the complex magnetic permeability of the samples (Equation (1)) over the frequency range of $1 \mathrm{kHz}$ to $2 \mathrm{MHz}$ was measured at room temperature, and the obtained results are presented in Figure 14 [106].

$$
\mu(f)=\mu^{\prime}(f)-i \mu^{\prime \prime}(f)
$$

where $\mu^{\prime}(f)$ is the real part; $\mu^{\prime \prime}(f)$ is the imaginary part.

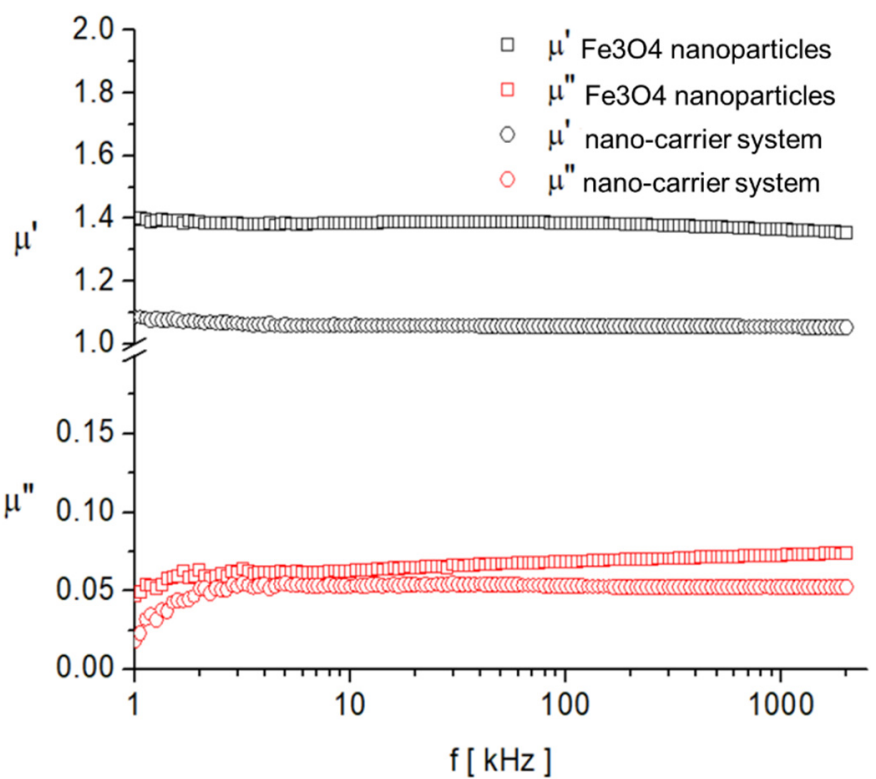

Figure 14. Frequency dependence of the real, $\mu^{\prime}$, and imaginary, $\mu^{\prime \prime}$, components of the complex magnetic permeability of samples.

In the investigated frequency range, there are no magnetic relaxation peaks that provide clues about the characteristic magnetization processes. However, given the small sizes of the particles and also the low value of the imaginary component of complex magnetic permeability, it can be assumed that the dominant magnetization mechanism is the Neel process, correlated with the rotation of the magnetic moment inside the particles by overcoming the magneto-crystalline anisotropy barrier [107,108].

The obtained results indicate that the nano-carrier system, wherein the selected ratio of plant:magnetic nanoparticles is 3:1, exhibits magnetic properties.

\section{Materials and Methods}

All used reagents were GC grade. Methanol and chloroform were purchased from VWR (Wien, Austria). The $\mathrm{Fe}_{3} \mathrm{O}_{4}$ nanoparticles (nanoparticle size: $23 \mathrm{~nm}$ ) were provided 
by the National Research and Development Institute for Non-Ferrous and Rare Metals, Pantelimon, Romania. The plant samples (whole plant) were collected in August 2020 from the area of Timis, Romania and were taxonomically authenticated at Victor Babes University of Medicine and Pharmacy, Timisoara, Romania. The plant samples were rapidly frozen in liquid nitrogen $\left(-194^{\circ} \mathrm{C}\right)$, ground and sieved to obtain a particle size lower than $0.5 \mathrm{~mm}$, and they were kept at $-80^{\circ} \mathrm{C}$ to avoid enzymatic conversion or metabolite degradation. For each analysis, $1.8 \mathrm{~g}$ of dried sample was subject to sonication extraction in $25 \mathrm{~mL}$ of solvent (methanol/chloroform $=1: 1$ ) for $25 \mathrm{~min}$ at $40{ }^{\circ} \mathrm{C}$ with a frequency of $50 \mathrm{kHz}$. The solution was concentrated using a rotavapor and the residue was dissolved in $\mathrm{MeOH}$. The extract was centrifuged, and the supernatant was filtered through a $0.2 \mu \mathrm{m}$ syringe filter and stored at $-18{ }^{\circ} \mathrm{C}$ until analysis.

\subsection{Nano-Carrier System Preparation}

For each analysis, $1.5 \mathrm{~g}$ of sample was prepared from dried herb (whole plant, ground and sieved to obtain a particle size lower than $0.5 \mathrm{~mm}$ ), and $\mathrm{Fe}_{3} \mathrm{O}_{4}$ nanoparticles were added (herb $/ \mathrm{Fe}_{3} \mathrm{O}_{4}$ nanoparticles $=3: 1$ ). The obtained mixture was subjected to micronization at room temperature for $5 \mathrm{~min}$.

\subsection{GC-MS Analysis}

Gas chromatography was carried out using ClarusSQ8 GC/MS (Perkin Elmer) apparatus with a ZB-AAA GC column $(10 \mathrm{~m} \times 0.25 \mathrm{~mm})$ (Phenomenex, Torrance, CA, USA); carrier gas, He; flow rate, $1 \mathrm{~mL} / \mathrm{min}$, following 3.3. GC-MS Separation Conditions (the standard conditions provided with the EZ: faast GC-MS free amino acids kit).

The oven temperature was $80{ }^{\circ} \mathrm{C}$ (held for $9 \mathrm{~min}$ ) to $220^{\circ} \mathrm{C}$ (held for $5 \mathrm{~min}$ ) at $320^{\circ} \mathrm{C} / \mathrm{min}$ (held for $10 \mathrm{~min}$ ); the equilibration time was $1 \mathrm{~min}$. The injection parameters were: split 1:5; $250{ }^{\circ} \mathrm{C} ; 2.5 \mu \mathrm{L}$. The carrier gas was helium; $1.1 \mathrm{~mL} / \mathrm{min} ; 110{ }^{\circ} \mathrm{C}$. The inlet pressure was $5.9 \mathrm{kPa} / \mathrm{min}$; the detector used was: MS; mode: Scan Transfer Line; temperature: $250{ }^{\circ} \mathrm{C}$; analyzer type: MS; electron energy: $70 \mathrm{eV}$.

\subsection{Mass Spectrometry}

MS experiments were conducted using EIS-QTOF-MS from Bruker Daltonics, Bremen, Germany. All mass spectra were acquired in the positive ion mode within a mass range of (100-3000) $\mathrm{m} / \mathrm{z}$, with a scan speed of $2.1 \mathrm{scans} / \mathrm{s}$. The source block temperature was kept at $80^{\circ} \mathrm{C}$. The reference provided a spectrum in positive ion mode with fair ionic coverage of the $m / z$ range scanned in full-scan MS. The resulting spectrum was a sum of scans over the total ion current (TIC) acquired at $25-85 \mathrm{eV}$ collision energy to provide the full set of diagnostic fragment ions.

\subsection{Identification of Metabolites}

The total ion current (TIC) and selected ion monitoring (SIM) values were compared with those from Phenomenex-EZ: faast amino acid analysis user guide and the results are presented in Table 1. The metabolites were identified via comparison of their mass spectra with those of the standard library NIST /NBS-3 (National Institute of Standards and Technology/National Bureau of Standards) spectral database, and the identified phytoconstituents are presented in Table 2.

\subsection{FT-IR Spectroscopy}

The FT-IR spectrum of the sample was recorded via KBr pellet using a Shimadzu Prestige-21 spectrometer in the range $400-4000 \mathrm{~cm}^{-1}$, with a resolution of $4 \mathrm{~cm}^{-1}$.

\subsection{XRD Spectroscopy}

The phase composition of the sample was determined via powder X-ray diffractometry (XRD) using monochromatic CuK $\alpha$ radiation $(\lambda=1.5406 \AA)$ on a Rigaku Ultima IV diffractometer equipped with a D/teX Ultra detector and operating at $40 \mathrm{kV}$ and $40 \mathrm{~mA}$. 
The analysis was performed in the $2 \theta$ range of $10-80^{\circ}$, with a scan speed of $5^{\circ} / \mathrm{min}$ and a step size of $0.01^{\circ} 2 \theta$. The average crystallite size was calculated using the whole pattern profile fitting method (WPPF). The XRD patterns were compared with those from the ICDD Powder Diffraction Database (ICDD file 04-015-9120).

\subsection{Scanning Electronic Microscopy (SEM)}

The SEM analyses were performed using an SEM-EDS system (QUANTA INSPECT F50) equipped with a field emission gun (FEG), $1.2 \mathrm{~nm}$ resolution and an energy dispersive X-ray spectrometer (EDS) with an MnK resolution of $133 \mathrm{eV}$.

\subsection{Magnetization Experiments}

The frequency dependence of the $\mathrm{Fe}_{3} \mathrm{O}_{4}$ nanoparticles and nano-carrier system was measured using an Agilent LCR-meter (E-4980A type) at room temperature over the frequency range ( $1 \mathrm{kHz}$ to $2 \mathrm{MHz}$ ) and various values of polarizing field. The duration of the measurement into a constant magnetic field over the entire frequency range was about $40 \mathrm{~s}$.

Complex magnetic susceptibility measurements were made using the short-circuited coaxial transmission line technique at different values of the polarizing field, $H$, over the range $0-170 \mathrm{kA} / \mathrm{m}$ and at the frequency range $(100 \mathrm{MHz}-6 \mathrm{GHz})$. The static magnetization measurements for the $\mathrm{Fe}_{3} \mathrm{O}_{4}$ nanoparticle sample and the nano-carrier system were performed using a ballistic galvanometer.

\section{Conclusions}

In the current study, the complete metabolite profiling of $A$. annua growing wild in Romania was accomplished. A total of 14 amino acids were identified for the for the first time in plant samples. The biological activities were discussed for each metabolite category. Furthermore, a simple and economical nano-carrier system was developed. A ratio of herb:magnetic $\mathrm{Fe}_{3} \mathrm{O}_{4}$ nanoparticles was used, which allowed for the synergic effect of $A$. annua bioactive compounds and its inorganic component properties to be taken advantage of. The morpho-structural characterization of the nano-carrier system was performed. In addition, the magnetic properties of the nano-carrier were evaluated. Further studies are necessary to investigate the biological properties and the bioavailability of the new nano-carrier system.

Author Contributions: Conception and design of study: A.-E.S. and I.G.; methodology: A.-E.S.; acquisition of data: C.M. and C.N.M.; analysis and interpretation of data: C.M. and C.N.M.; writingoriginal draft preparation: I.O.-F.G.; writing-review and editing: A.-E.S. investigation: I.O.-F.G. and C.V.I.F. All authors have read and agreed to the published version of the manuscript.

Funding: Grant CNFIS-FDl-2021-0414, titled: "Centers of excellence with the support of UVT researchers" (Centre de excelenţă prin sprijinul cercetatorilor din UVT).

Institutional Review Board Statement: Not applicable.

Informed Consent Statement: Not applicable.

Data Availability Statement: All data are contained within the article.

Acknowledgments: The National Center for Micro and Nanomaterials (the center is part of the Department of Science and Engineering of Oxide and Nanomaterials Materials of the Faculty of Applied Chemistry and Materials Science of the Politehnica University of Bucharest). Grant CNFISFD1-2021-0414, titled: "Centers of excellence with the support of UVT researchers" (Centre de excelenţă prin sprijinul cercetatorilor din UVT).

Conflicts of Interest: The authors declare no conflict of interest.

\section{References}

1. Dihoru, G.; Boruz, V. The List of Main Spontaneous Medicinal Plants from Romania, Annals of the University of Craiova-Agriculture, Montanology, Cadastre Series; XLIV 328; University of Craiova: Craiova, Romania, 2014. 
2. Pantu Zach, C. Plantele Cunoscute de Poporul Roman-Vocabular Botanic Cuprinzand Numirile Romane, Franceze, Germane si Stiintifice; Institutul de Arte Grafice, Ed.; Minerva: Bucuresti, Romania, 1906.

3. Cântar, I.C.; Dincă, M. Curiosities about the Artemisia Collections from "Alexandru Beldie" Herbarium, Annals of the University of Craiova-Agriculture, Montanology, Cadastre Series; XLIX; University of Craiova: Craiova, Romania, 2019.

4. Clinciu Radu, R.A.; Zaharia, M.S.; Lungoci, C.; Vodă, A.D.; Nițu, S.; Robu, T. Studies Regarding the Growth Dynamics of Some Species from Artemisia Genus, Annals of the University of Oradea. Fascicle 2017, 29, 29-34.

5. Stan, R.L.; Sevastre, B.; Ionescu, C.; Olah, N.K.; Vicas, L.G.; Páll, E.; Moisa, C.; Hanganu, D.; Sevastre-Berghian, A.C.; Andrei, S.; et al. Artemisia Annua L. extract: A new phytoproduct with sod-like and antitumour activity. Farmacia 2020, 68, 812-821. [CrossRef]

6. Weathers, P.J.; Arsenault, P.R.; Covello, P.S.; McMickle, A.; Teoh, K.H.; Reed, D.W. Artemisinin production in Artemisia annua: Studies in planta and results of a novel delivery method for treating malaria and other neglected diseases. Phytochem. Rev. 2011, 10, 173-183. [CrossRef] [PubMed]

7. Mamatova, A.S.; Korona-Glowniak, I.; Skalicka-Woźniak, K.; Józefczyk, A.; Wojtanowski, K.K.; Baj, T.; Sakipova, Z.B.; Malm, A. Phytochemical composition of wormwood (Artemisia gmelinii) extracts in respect of their antimicrobial activity. BMC Complement. Altern. Med. 2019, 19, 288. [CrossRef] [PubMed]

8. Deb, M.; Kumar, D. Chemical composition and bioactivity of the essential oils derived from Artemisia Annua against the red flour beetle. Biosci. Biotech. Res. Asia 2019, 16, 463-476. [CrossRef]

9. Mesa, L.E.; Lutgen, P.; Velez, I.D.; Segura, A.M.; Robledo, S.M. Artemisia annua L., Potential source of molecules with pharmacological activity in human diseases. Am. J. Phytomed. Clin. Ther. 2015, 3, 436-450.

10. Iqbal, S.; Younas, U.; Chan, K.W.; Zia-Ul-Haq, M.; Ismail, M. Chemical composition of Artemisia annua L. leaves and antioxidant potential of extracts as a function of extraction solvents. Molecules 2012, 17, 6020-6032.

11. Nigam, M.; Atanassova, M.; Mishra, A.P.; Pezzani, R.; Devkota, H.P.; Plygun, S.; Salehi, B.; Setzer, W.N.; Sharifi-Rad, J. Bioactive compounds and health benefits of Artemisia species. Nat. Prod. Commun. 2019, 14, 1934578X19850354.

12. Sadiq, A.; Hayat, M.Q.; Ashraf, M. Ethnopharmacology of Artemisia annua L.: A Review. In Artemisia Annua-Pharmacology and Biotechnology; Aftab, T., Ferreira, J.F.S., Khan, M.M.A., Naeem, M., Eds.; Springer: Berlin/Heidelberg, Germany, 2014 ; pp. 9-25.

13. Badshah, S.L.; Ullah, A.; Ahmad, N.; Almarhoon, Z.M.; Mabkhot, Y. Increasing the strength and production of artemisinin and its derivatives. Molecules 2018, 23, 100. [CrossRef]

14. Septembre-Malaterre, A.; Lalarizo Rakoto, M.; Marodon, C.; Bedoui, Y.; Nakab, J.; Simon, E.; Hoarau, L.; Savriama, S.; Strasberg, D.; Guiraud, P.; et al. Artemisia annua, a traditional plant brought to light. Int. J. Mol. Sci. 2020, 21, 4986. [CrossRef]

15. Farid-ul-Haq Muhammad Haseeb, M.T.; Hussain, M.A.; Ashraf, M.U.; Naeem-ul-Hassan, M.; Hussain, S.Z.; Hussain, I. A smart drug delivery system based on Artemisia vulgaris hydrogel: Design, on-off switching, and real-time swelling, transit detection, and mechanistic studies. J. Drug Deliv. Sci. Technol. 2020, 58, 101795. [CrossRef]

16. Li, Z.; Li, Q.; Wu, J.; Wang, M.; Yu, J. Artemisinin and its derivatives as a repurposing anticancer agent: What else do we need to do? Molecules 2016, 21, 1331. [CrossRef] [PubMed]

17. Shahrajabian, M.H.; Sun, W.; Cheng, Q. Exploring Artemisia annua L., artemisinin and its derivatives, from traditional chinese wonder medicinal science. Not. Bot. Horti Agrobot. Cluj-Napoca 2020, 48, 1719-1741. [CrossRef]

18. Bilia, A.R.; Santomauro, F.; Sacco, C.; Bergonzi, M.C.; Donato, R. Essential oil of Artemisia annua L.: An Extraordinary component with numerous antimicrobial properties. Evid.-Based Complement. Altern. Med. 2014, 2014, 159819. [CrossRef]

19. Bora, K.S.; Sharma, A. The genus Artemisia: A comprehensive review. Pharm. Biol. 2011, 49, 101-109. [CrossRef] [PubMed]

20. Das, S. Artemisia annua (Qinghao): A pharmacological review. Int. J. Pharm. Sci. Res. 2012, 3, 4573-4577.

21. Numonov, S.; Sharopov, F.; Salimov, A.; Sukhrobov, P.; Atolikshoeva, S.; Safarzoda, R.; Habasi, M.; Aisa, H.A. Assessment of artemisinin contents in selected Artemisia Species from Tajikistan (Central Asia). Medicines 2019, 6, 23. [CrossRef]

22. Abad, M.J.; Bedoya, L.M.; Apaza, L.; Bermejo, P. The Artemisia L. genus: A review of bioactive essential oils. Molecules 2012, 17, 2542-2566. [CrossRef]

23. Lang, S.J.; Schmiech, M.; Hafner, S.; Paetz, C.; Steinborn, C.; Huber, R.; El Gaafary, M.; Werner, K.; Schmidt, C.Q.; Syrovets, T.; et al. Antitumor activity of an Artemisia annua herbal preparation and identification of active ingredients. Phytomedicine 2019, 62, 152962. [CrossRef]

24. World Malaria Report. In 20 Years of Global Progress and Challenges; World Health Organization: Geneva, Switzerland, 2020; ISBN 978-92-4-001579-1.

25. Charlie-Silva, I.; Fernandes Fraceto, L.; Ferreira Silva de Melo, N. Progress in nano-drug delivery of artemisinin and its derivatives: Towards to use in immunomodulatory approaches. Artif. Cells Nanomed. Biotechnol. 2018, 46, S611-S620. [CrossRef]

26. Zhao, Q.; Luan, X.; Zheng, M.; Tian, X.H.; Zhao, J.; Zhang, W.D.; Ma, B.L. Synergistic mechanisms of constituents in herbal extracts during intestinal absorption: Focus on natural occurring nanoparticles. Pharmaceutics 2020, 12, 128. [CrossRef]

27. Hussein, R.A.; El-Anssary, A.A. Plants Secondary Metabolites: The Key Drivers of the Pharmacological Actions of Medicinal Plants, Herbal Medicine; Builders, P.F., Ed.; IntechOpen Limited: London, UK, 2018.

28. Tzenkova, R.; Kamenarska, Z.; Draganov, A.; Atanassov, A. Composition of Artemisia annua essential oil obtained from species gowing wild in Bulgaria. Biotechnol. Biotechnol. Equip. 2010, 24, 1833-1835. [CrossRef]

29. Albaugh, V.L.; Pinzon-Guzman, C.; Barbul, A. Arginine-Dual roles as an onconutrient and immunonutrient. J. Surg. Oncol. 2017, 115, 273-280. [CrossRef] [PubMed] 
30. Sun-Hee, K.; Roszik, J.; Grimm, E.A.; Ekmekcioglu, S. Impact of L-Arginine metabolism on immune response and anticancer immunotherapy. Front. Oncol. 2018, 8, 67.

31. Chiangjong, W.; Chutipongtanate, S.; Hongeng, S. Anticancer peptide: Physicochemical property, functional aspect and trend in clinical application (Review). Int. J. Oncol. 2020, 57, 678-696. [CrossRef] [PubMed]

32. Ochkur, A.V.; Kovaleva, A.M.; Kolesnik, Y.S. Amino-acid composition of subgenus Artemisia Herbs. Chem. Nat. Compd. 2013, 49, 589-591. [CrossRef]

33. Berechet, M.D.; Stelescu, M.D.; Manaila, E.; Craciun, G. Chemical composition of the essential oil of Artemisia absinthium from Romania. Rev. Chim. 2015, 66, 1814-1818.

34. Moacă, E.A.; Pavel, I.Z.; Danciu, C.; Crăiniceanu, Z.; Minda, D.; Ardelean, F.; Antal, D.S.; Ghiulai, R.; Cioca, A.; Derban, M.; et al. Romanian wormwood (Artemisia absinthium L.): Physicochemical and nutraceutical screening. Molecules 2019, $24,3087$. [CrossRef]

35. Ivanescu, B.; Vlase, L.; Corciova, A.; Lazar, M.I. Artemisinin evaluation in Romanian Artemisia annua wild plants using a new HPLC/MS method. Nat. Prod. Res. 2011, 25, 716-722. [CrossRef]

36. Marinas, I.C.; Oprea, E.; Chifiriuc, M.C.; Badea, I.A.; Buleandra, M.; Lazar, V. Chemical composition and antipathogenic activity of Artemisia annua essential oil from Romania. Chem. Biodivers. 2015, 12, 1554-1564. [CrossRef]

37. Toth, E.T.; Dezso, A.C.; Kapas, A.; Pako, J.; Ichim, M.C. Comparison of chemical composition of Artemisia annua volatile oil from Romania, Chemical composition and antipathogenic activity of Artemisia annua essential oil from Romania. Planta Med. 2011, 77, PL91. [CrossRef]

38. Ruperti-Repilado, F.J.; Haefliger, S.; Rehm, S.; Zweier, M.; Rentsch, K.M.; Blum, J.; Jetter, A.; Heim MLeuppi-Taegtmeyer, A.; Terracciano, L.; Bernsmeier, C. Danger of herbal tea: A case of acute cholestatic hepatitis due to Artemisia annua tea. Front. Med. 2019, 6, 221. [CrossRef] [PubMed]

39. Alsanad, S.; Howard, R.; Williamson, E. An assessment of the impact of herb-drug combinations used by cancer patients. BMC Complementary Altern. Med. 2016, 16, 393. [CrossRef]

40. Fouad, D.; Bachra, Y.; Ayoub, G.; Ouaket, A.; Bennamara, A.; Knouzi, N.; Berrada, M. A Novel Drug Delivery System Based on Nanoparticles of Magnetite Fe3O4 Embedded in an Auto Cross-Linked Chitosan [Online First]; IntechOpen Limited: London, UK, 2020

41. Rahman, H.S.; Othman, H.H.; Hammadi, N.I.; Yeap, S.K.; Amin, K.M.; Abdul Samad, N.; Alitheen, N.B. Novel drug delivery systems for loading of natural plant extracts and their biomedical applications. Int. J. Nanomed. 2020, 15, 2439-2483. [CrossRef]

42. Mohammadi, M.; Pourseyed Aghaei, F. Magnetite $\mathrm{Fe}_{3} \mathrm{O}_{4}$ surface as an effective drug delivery system for cancer treatment drugs: Density functional theory study. J. Biomol. Struct. Dyn. 2021, 39, 2798-2805. [CrossRef]

43. Gómez-Sotomayor, R.; Ahualli, S.; Viota, J.L.; Rudzka, K.; Delgado, Á.V. Iron/Magnetite nano-particles as magnetic delivery systems for antitumor drugs. J. Nanosci. Nanotechnol. 2015, 15, 3507-3514. [CrossRef]

44. Socoliuc, V.; Peddis, D.; Petrenko, V.I.; Avdeev, M.V.; Susan-Resiga, D.; Szabó, T.; Turcu, R.; Tombácz, E.; Vékás, L. Magnetic nanoparticle systems for nanomedicine-A materials science perspective. Magnetochemistry 2020, 6, 2. [CrossRef]

45. Aderibigbe, B.A. Design of drug delivery systems containing artemisinin and its derivatives. Molecules 2017, 22, 323. [CrossRef]

46. Elfawal, M.A.; Towler, M.J.; Reich, N.G.; Golenbock, D.; Weathers, P.J.; Rich, S.M. Dried whole plant Artemisia annua as an antimalarial therapy. PLOS ONE 2012, 7, e52746. [CrossRef]

47. Chen, J.; Guo, Z.; Wang, H.-B.; Zhou, J.-J.; Zhang, W.-J.; Chen, Q.-W. Multifunctional mesoporous nanoparticles as pH-responsive $\mathrm{Fe}^{2+}$ reservoirs and artemisinin vehicles for synergistic inhibition of tumor growth. Biomaterials 2014, 35, 6498-6507. [CrossRef]

48. Wang, Y.; Han, Y.; Yang, Y.; Yang, J.; Guo, X.; Zhang, J.; Pan, L.; Xia, G.; Chen, B. Effect of interaction of magnetic nanoparticles of $\mathrm{Fe}_{3} \mathrm{O}_{4}$ and artesunate on apoptosis of $\mathrm{K} 562$ cells. Int. J. Nanomed. 2011, 6, 1185-1192.

49. Akbari, M.; Mora, R.; Maaza, M. First principle study of silver nanoparticle interactions with antimalarial drugs extracted from Artemisia annua plant. J. Nanopart. Res. 2020, 22, 331. [CrossRef] [PubMed]

50. Chen, G.; Wang, S.; Huang, X.; Hong, J.; Du, L.; Zhang, L.; Ye, L. Environmental factors affecting growth and development of Banlangen (Radix Isatidis) in China. Afr. J. Plant Sci. 2015, 9, 421-426.

51. Pang, Z.; Chen, J.; Wang, T.; Gao, C.; Li, Z.; Guo, L.; Xu, J.; Cheng, Y. Linking plant secondary metabolites and plant microbiomes: A Review. Front. Plant Sci. 2021, 12, 621276. [CrossRef] [PubMed]

52. Zhang, X.; Zhao, Y.; Guo, L.; Qiu, Z.; Huang, L.; Qu, X. Differences in chemical constituents of Artemisia annua L from different geographical regions in China. PLoS ONE 2017, 12, e0183047. [CrossRef]

53. Adams, R.P. Identification of Essential Oil Components by Gas Chromatography/Mass Spectroscopy; Allured Publishing Corporation: Carol Stream, IL, USA, 2007.

54. Segneanu, A.-E.; Grozescu, I.; Cziple, F.; Berki, D.; Damian, D.; Niculite, C.M.; Florea, A.; Leabu, M. Helleborus purpurascensAmino acid and peptide analysis linked to the chemical and antiproliferative properties of the extracted compounds. Molecules 2015, 20, 22170-22187. [CrossRef] [PubMed]

55. Phenomenex, E.Z. Faast-Easy Fast Amino Acid Sampling Testing Kit-User Guide; Torrance, CA, USA; Available online: http: / / www.phenomenex.com (accessed on 12 October 2021).

56. Czechowski, T.; Rinaldi, M.A.; Toyin, F.M.; Van Veelen, M.; Larson, T.R.; Thilo, W.; Rathbone, D.A.; David, H.; Horrocks, P.; Graham, I.A. Flavonoid versus artemisinin anti-malarial activity in Artemisia annua whole-leaf extracts. Front. Plant Sci. 2019, 10, 984. [CrossRef] [PubMed] 
57. Ferreira, J.F.S.; Luthria, D.L.; Sasaki, T.; Heyerick, A. Flavonoids from Artemisia annua L. as antioxidants and their potential synergism with artemisinin against malaria and cancer. Molecules 2010, 15, 3135-3170. [PubMed]

58. Silveira de Almeida, L.M.; Soares Aleixo de Carvalho, L.; Gazolla, M.C.; Silva Pinto, P.L.; Nascimento da Silva, M.P.; de Moraes, J.; Da Silva Filho, A.A. Flavonoids and sesquiterpene lactones from Artemisia absinthium and Tanacetum parthenium against Schistosoma mansoni worms. Evid.-Based Complement. Altern. Med. 2016, 2016, 9521349.

59. Baraldi, R.; Isacchi, B.; Predieri, S.; Marconi, G.; Vincieri, F.F.; Bilia, A.R. Distribution of artemisinin and bioactive flavonoids from Artemisia annua L. during plant growth. Biochem. Syst. Ecol. 2008, 36, 340-348. [CrossRef]

60. Chu, Y.; Wang, H.; Chen, J.; Hou, Y. New sesquiterpene and polymethoxy-flavonoids from Artemisia annua L. Phcog. Mag. 2014, 10, 213-216. [CrossRef]

61. Brisibe, E.A.; Umoren, E.U.; Brisibe, F.; Magalhäes, P.M.; Ferreira, J.F.S.; Luthria, D.; Wu, X.; Prior, R. Nutritional characterisation and antioxidant capacity of different tissues of Artemisia annua L. Food Chem. 2009, 115, 1240-1246. [CrossRef]

62. Sharopov, F.S.; Salimov, A.; Numonov, S.; Safomuddin, A.; Bakri, M.; Salimov, T.; Setzer, W.N.; Habasi, M. Chemical composition, antioxidant, and antimicrobial activities of the essential oils from Artemisia annua L. growing wild in Tajikistan. Nat. Prod. Commun. 2020, 15, 1934578X20927814.

63. Bhakuni, R.; Jain, D.; Sharma, R.; Kumar, S. Secondary metabolites of Artemisia annua and their biological activity. Curr. Sci. 2001, 80, 35-48.

64. Yang, S. Phytochemical Studies of Artemisia annua L. Ph.D. Thesis, Department of Pharmacognosy, The School of Pharmacy, The University of London, ProQuest LLC, London, UK, 2017.

65. Trendafilova, A.; Moujir, L.M.; Sousa, P.M.C.; Seca, A.M.L. Research advances on health effects of edible Artemisia species and some sesquiterpene lactones constituents. Foods 2021, 10, 65. [CrossRef] [PubMed]

66. Dawood, H.M.; Shawky, E.; Hammoda, H.M.; Metwally, A.M.; Ibrahim, R.S. Chemical constituents from Artemisia annua and Vitex agnus-castus as new aromatase inhibitors: In-vitro and in-silico studies. J. Mex. Chem. Soc. 2020, 64, 316-326. [CrossRef]

67. Brown, G.D. The biosynthesis of artemisinin (Qinghaosu) and the phytochemistry of Artemisia annua L. (Qinghao). Molecules 2010, 15, 7603-7698.

68. Nikolova, M.; Gevrenova, R.; Ivancheva, S. High-performance liquid chromatographic separation of surface flavonoid aglycones in Artemisia annua L. and Artemisia vulgaris L. J. Serb. Chem. Soc. 2004, 69, 571-574. [CrossRef]

69. Nikolova, M. Infraspecific variability in the flavonoid composition of Artemisia vulgaris L. Acta Bot. Croat. 2006, 65, 13-18.

70. Valant-Vetscheraa, K.M.; Wollenweberb, E. Flavonoid aglycones from the leaf surfaces of some Artemisia spp. (CompositaeAnthemideae). Z. Naturforsch. 1995, 50, 353-357. [CrossRef]

71. Rodríguez, E.; Carman, N.J.; Vander Velde, G.; McReynolds, J.H.; Mabry, T.J.; Irwin, M.A.; Geissman, T.A. Methoxylated flavonoids from Artemisia. Phytochemistry 1972, 11, 3509-3514. [CrossRef]

72. Chougouo, R.D.; Nguekeu, Y.M.; Dzoyem, J.P.; Awouafack, M.D.; Kouamouo, J.; Tane, P.; McGaw, L.J.; Eloff, J.N. Antiinflammatory and acetylcholinesterase activity of extract, fractions and five compounds isolated from the leaves and twigs of Artemisia annua growing in Cameroon. Springerplus 2016, 5, 1525. [CrossRef] [PubMed]

73. Lieu, E.L.; Nguyen, T.; Rhyne, S.; Kim, J. Amino acids in cancer. Exp. Mol. Med. 2020, 52, 15-30. [CrossRef] [PubMed]

74. Cox-Georgian, D.; Ramadoss, N.; Dona, C.; Basu, C. Therapeutic and medicinal uses of terpenes. Med. Plants 2019, 12, 333-359.

75. Fongang Fotsing, Y.S.; Bankeu Kezetas, J.J. Terpenoids as important bioactive constituents of essential oils. In Essential OilsBioactive Compounds, New Perspectives and Application; Santana de Oliveira, M., Almeida da Costa, W., Gomes Silva, S., Eds.; IntechOpen Limited: London, UK, 2020; ISBN 978-1-83962-698-2.

76. Jahangeer, M.; Fatima, R.; Ashiq, M. Therapeutic and biomedical potentialities of terpenoids-A Review. J. Pure Appl. Microbiol. 2021, 15, 471-483. [CrossRef]

77. Küpeli Akkol, E.; Genç, Y.; Karpuz, B.; Sobarzo-Sánchez, E.; Capasso, R. Coumarins and coumarin-related compounds in pharmacotherapy of cancer. Cancers 2020, 12, 1959. [CrossRef]

78. João Matos, M.; Santana, L.; Uriarte, E.; Abreu, O.A.; Molina, E.; Guardado Yordi, E. Coumarins—An Important Class of Phytochemicals in Phytochemicals-Isolation, Characterisation and Role in Human Health; Venket Rao, A., Rao, L.G., Eds.; IntechOpen Limited: London, UK, 2015.

79. Kozłowska, A.; Szostak-Wegierek, D. Flavonoids-food sources and health benefits. Rocz. Panstw. Zakl. Hig. 2014, $65,79-85$.

80. Panche, A.N.; Diwan, A.D.; Chandra, S.R. Flavonoids: An overview. J. Nutr. Sci. 2016, 5, e47. [CrossRef]

81. Kozłowska, A.; Szostak-Węgierek, D. Flavonoids-food sources, health benefits, and mechanisms involved. In Bioactive Molecules in Food; Mérillon, J.M., Ramawat, K., Eds.; Reference Series in Phytochemistry; Springer: Cham, Switzerland, 2018.

82. Kumar, N.; Goel, N. Phenolic acids: Natural versatile molecules with promising therapeutic applications. Biotechnol. Rep. 2019, 24, e00370. [CrossRef]

83. Minatel, I.O.; Vanz Borges, C.; Ferreira, M.I.; Gomez, H.A.G.; Chen, C.Y.O. Pace Pereira Lima G., Phenolic compounds: Functional properties, impact of processing and bioavailability. In Phenolic Compounds-Biological Activity; Soto-Hernandez, M., PalmaTenango, M., del Rosario Garcia-Mateos, M., Eds.; IntechOpen Limited: London, UK, 2017.

84. Salehi, B.; Quispe, C.; Sharifi-Rad, J.; Cruz-Martins, N.; Nigam, M.; Mishra, A.P.; Konovalov, D.A.; Orobinskaya, V.; Abu-Reidah, I.M.; Zam, W.; et al. Phytosterols: From preclinical evidence to potential clinical applications. Front. Pharmacol. 2021, 11, 599959. [CrossRef] 
85. Nagy, K.; Tiuca, I.-D. Importance of Fatty Acids in Physiopathology of Human Body in Fatty Acids; Catala, A., Ed.; IntechOpen Limited: London, UK, 2017.

86. Kilcoyne, M.; Joshi, L. Carbohydrates in therapeutics. Cardiovasc. Hematol. Agents Med. Chem. 2007, 5, 186-197. [CrossRef]

87. Singh, D.; Rajput, A.; Bhatia, A.; Kumar, A.; Kaur, H.; Sharma, P.; Kaur, P.; Singh, S.; Attri, S.; Buttar, H.; et al. Plant-based polysaccharides and their health functions. Funct. Foods Health Dis. 2021, 11, 179-200.

88. Dhama, K.; Karthik, K.; Khandia, R.; Munjal, A.; Tiwari, R.; Rana, R.; Khurana, S.K.; Ullah, S.; Khan, R.U.; Alagawany, M.; et al . Medicinal and therapeutic potential of herbs and plant metabolites/Extracts countering viral pathogens-Current knowledge and future prospects. Curr. Drug Metab. 2018, 19, 236-263. [CrossRef] [PubMed]

89. Khan, H.; Saeedi, M.; Nabavi, S.; Mubarak, M.; Bishayee, A. Glycosides from medicinal plants as potential anticancer agents: Emerging trends towards future drugs. Curr. Med. Chem. 2018, 26, 2389-2406. [CrossRef]

90. Mohammed, L.; Gomaa, H.G.; Ragab, D.; Zhu, J. Magnetic nanoparticles for environmental and biomedical applications: A review. Particuology 2017, 30,1-14. [CrossRef]

91. Wahajuddin Arora, S. Superparamagnetic iron oxide nanoparticles: Magnetic nanoplatforms as drug carriers. Int. J. Nanomed. 2012, 7, 3445-3471. [CrossRef]

92. Nunes Oliveira, R.; Cordeiro Mancini, M.; Salles de Oliveira, F.C.; Marques Passos, T.; Quilty, B.; da Silva Moreira Thiré, R.M.; McGuinness, G.B. FTIR analysis and quantification of phenols and flavonoids of five commercially available plants extracts used in wound healing. Matéria 2016, 21, 767-779.

93. Kristoffersen, K.A.; van Amerongen, A.; Böcker, U.; Lindberg, D.; Wubshet, S.G.; de Vogel-van den Bosch, H.; Horn, S.J.; Afseth, N.K. Fourier-transform infrared spectroscopy for monitoring proteolytic reactions using dry-films treated with trifluoroacetic acid. Sci. Rep. 2020, 10, 7844. [CrossRef]

94. Ercioglu, E.; Velioglu, H.M.; Boyaci, I.H. Determination of terpenoid contents of aromatic plants using NIRS. Talanta 2018, 178, 716-721. [CrossRef]

95. Mawa, S.; Jantan, I.; Husain, K. Isolation of terpenoids from the stem of Ficus aurantiaca Griff and their effects on reactive oxygen species production and chemotactic activity of neutrophils. Molecules 2016, 21, 9. [CrossRef]

96. Sai Prakash Chaturvedula, V.; Mubarak, C.; Prakash, I. IR spectral analysis of diterpene glycosides isolated from Stevia rebaudiana. Food Nutr. Sci. 2012, 3, 1467-1471.

97. Forfang, K.; Zimmermann, B.; Kosa, G.; Kohler, A.; Shapaval, V. FTIR Spectroscopy for evaluation and monitoring of lipid extraction efficiency for oleaginous fungi. PLoS ONE 2017, 12, e0170611. [CrossRef] [PubMed]

98. Mehrotra, R. Infrared spectroscopy, gas chromatography/Infrared in food analysis. In Encyclopedia of Analytical Chemistry; Meyers, R.A., Ed.; Wiley: Hoboken, NJ, USA, 2006; ISBN 978047197670.

99. Iranshahi, M.; Emami, S.A.; Mahmoud-Soltani, M. Detection of sesquiterpene lactones in ten Artemisia species population of Khorasan Provinces. Iran. J. Basic Med Sci. 2007, 10, 183-188.

100. Limem, S.; Maazaoui, R.; Mani Kongnine, D.; Mokhtar, F.; Karmous, T. Preliminary identification of Citrullus colocynthis from Togo by FT-IR and Raman Spectroscopy. Int. J. Adv. Res. 2015, 3, 354-360.

101. Segneanu, A.E.; Gozescu, I.; Dabici, A.; Sfirloaga, P.; Szabadai, Z. Organic Compounds FT-IR Spectroscopy in Macro to Nano Spectroscopy; Uddin, J., Ed.; InTechOpen: Rijeka, Croatia, 2012; ISBN 978-953-51-0664-7.

102. Monallem-Bahont, M.; Bertrand, S.; Pena, O. Synthesis and characterization of Zn1-xNixFe2O4 spinels prepared by a citrate precursor. J. Solid State Chem. 2005, 178, 1080-1086. [CrossRef]

103. Ahn, Y.; Choi, E.J.; Kim, E.H. Superparamagnetic relaxation in cobalt ferrite nano-particles synthesized from hydroxide carbonate precursors. Rev. Adv. Mater. Sci. 2003, 5, 477-480.

104. Naseri, M.G.; Saion, E.B.; Hashim, M.; Shaari, A.H.; Ahangar, H.A. Synthesis and characterization of zinc ferrite nanoparticles by a thermal treatment method. Solid State Commun. 2011, 151, 1031-1035. [CrossRef]

105. Mihalca, I.; Ercuta, A. Structural relaxation in Fe70Cr10.5P11.5Mn1.5C6.5 amorphous alloys. J. Optoelectron. Adv. Mater. 2003, 5, 245-249.

106. Marin, C.N.; Fannin, P.C.; Malaescu, I. Time solved susceptibility spectra of magnetic fluids. J. Magn. Magn. Mater. 2015, 388, 45-48. [CrossRef]

107. Fannin, P.C.; Marin, C.N. Determination of the Landau-Lifshitz damping parameter by means of complex susceptibility measurements. J. Magn. Magn. Mater. 2006, 299, 425-429. [CrossRef]

108. Mălăescu, I.; Marin, C.N. Study of magnetic fluids by means of magnetic spectroscopy. Phys. B Condens. Matter 2005, 365, 134-140. 